\title{
Design Verification and Performance Evaluation of an Enhanced Wideband CDMA Receiver Using Channel Measurements
}

\author{
Karim Cheikhrouhou, ${ }^{1}$ Sofiène Affes, ${ }^{1}$ Ahmed Elderini,, ${ }^{1}$ Besma Smida, ${ }^{1}$ Paul Mermelstein, ${ }^{1}$ \\ Belhassen Sultana, ${ }^{2}$ and Venkatesh Sampath ${ }^{3}$ \\ ${ }^{1}$ INRS-EMT, Université du Québec, 800 de la Gauchetière Ouest, bureau 6900, Montreal, QC, Canada H5A 1 K6 \\ Emails: cheikhro@emt.inrs.ca, sofiene.affes@emt.inrs.ca,elderini@emt.inrs.ca,smida@emt.inrs.ca,mermel@emt.inrs.ca \\ ${ }^{2}$ Microcell Solutions, Inc., 771 Gougeon, St. Laurent, QC, Canada H4T 2B4 \\ Email: belhassen.sultana@fidomobile.ca \\ ${ }^{3}$ Flarion Technologies, Inc., Bedminster, NJ 07921, USA \\ Email:v.sampath@flarion.com
}

Received 15 March 2004; Revised 16 August 2004

\begin{abstract}
The spatio-temporal array receiver (STAR) decomposes generic wideband CDMA channel responses across various parameter dimensions (e.g., time delays, multipath components, etc.) and extracts the associated time-varying parameters (i.e., analysis) before reconstructing the channel (i.e., synthesis) with increased accuracy. This work verifies the channel analysis/synthesis design of STAR by illustrating its capability to extract accurately the channel parameters (time delays and drifts, carrier frequency offsets, Doppler spread, etc.) from measured data and to adapt online to their observed time evolution in real-world propagation conditions. We also verify the performance of STAR by comparing the results achieved with generic and measured channels for an average multipath power profile of $[0,-4,-8] \mathrm{dB}$ and a vehicular speed below $30 \mathrm{~km} / \mathrm{h}$. The results suggest that losses due to operations with real channels are only $1 \mathrm{~dB}$ in SNR and 20-30\% in capacity with DBPSK and single transmit and receive antennas. The corresponding SNR threshold for operation with real channels is about $5 \mathrm{~dB}$.
\end{abstract}

Keywords and phrases: wideband CDMA, design verification, performance evaluation, channel measurements, analysis/synthesis, dynamic channel characterization.

\section{INTRODUCTION}

New technical results in transceiver design are expected to influence significantly the organization of future thirdgeneration $(3 \mathrm{G})$ wireless networks and beyond $(3 \mathrm{G}+)$ to support multimedia data communication and wireless access to the Internet. (We use 3G and 3G+ to refer to "IMT2000" (International Mobile Telecommunications-2000) and to "systems beyond IMT-2000," respectively. Note that some already refer to "systems beyond IMT-2000" as fourthgeneration $(4 \mathrm{G})$ systems.) Current second-generation $(2 \mathrm{G})$ systems are generally limited to less than $100 \mathrm{kbps}$ per user. Next-generation systems will soon allow up to $2 \mathrm{Mbps}$ with current $3 \mathrm{G}$ deployment and up to $10-20 \mathrm{Mbps}$ with 3G+ deployment by 2010. Without cost-effective advanced

This is an open access article distributed under the Creative Commons Attribution License, which permits unrestricted use, distribution, and reproduction in any medium, provided the original work is properly cited. transceiver designs that integrate new features such as enhanced channel synchronization and identification, multiuser detection, MIMO, and so forth, transmissions at such high rates may not be achievable.

Motivated by the need for increased bandwidth efficiencies, we have recently developed a new spatio-temporal array receiver (STAR) $[1,2]$ that achieves accurate and fast temporal synchronization, channel identification, and efficient signal combining with significant gains in performance over RAKE-type receivers [2,3]. More recently, we upgraded STAR to integrate space-time multiuser detection based on interference subspace rejection (ISR) [4], carrier frequency offset (CFO) recovery (CFOR) $[5,6,7]$, and multiple-input multiple-output (MIMO) high-speed transmissions on the downlink [8]. All these significant enhancements exploit the powerful dynamic channel parameter extraction capabilities of STAR based on a priori known generic models for these parameters. Indeed, STAR applies an "analysis/synthesis" principle where it decomposes the channel 
over various channel parameter dimensions (e.g., time delays, shaping pulse, Rayleigh fades, etc.), estimates those time-varying parameters (i.e., analysis), then uses them to reconstruct the channel (i.e., synthesis) with increased accuracy $[1,2,4]$. Verification of the software design and evaluation of STAR's performance using real-world channel measurements are important steps toward practical receiver implementations.

In this work, we verify the analysis/synthesis-based design of STAR by assessing its ability to extract online the channel parameters (time delays and drifts, multipath components and average multipath power profiles, CFOs, and Doppler spread or speed) from $5 \mathrm{MHz}$ radio channels measured at $2 \mathrm{GHz}$. We verify its performance by comparing the results obtained with measured channels to those obtained with generic channel models tuned with the extracted parameters both at the link and system levels. In the process, we take advantage of the unique parameter extraction capability of STAR to investigate the dynamic characteristics of the wideband radio channel at $2 \mathrm{GHz}$ (e.g., time-evolution of the time delays, CFO, Doppler spread, etc.). Such a dynamic analysis is in contrast with the traditional static analyses, which are very useful but are based only on statistical measurements of the channel $[9,10,11]$. In the recent context where adaptive receivers find very efficient use in wireless communications, it becomes essential not only to estimate the statistics of the wireless channel, but also to have a very thorough understanding of the time realizations of its parameters and from there identify ways to reduce the imperfection of their generic time-evolution models on which heavily depends the performance of adaptive $3 \mathrm{G} / 3 \mathrm{G}+$ receivers such as STAR. Computational simplifications may be attained if the processing rate of these adaptive receivers match the time variations of the channel parameters. This could eventually lead to a reduction in the complexity of $3 \mathrm{G} / 3 \mathrm{G}+$ receivers in the future.

The paper is organized as follows. In Section 2, we explain the analysis/synthesis-based data model and receiver design in STAR. In Section 3, we provide brief descriptions of the verification approach and methodology adopted, the database of real-world channel measurements, and the link/system-level simulator setup. In Section 4, we report on the design verification results by illustrating the capacity of STAR of instantly extracting the channel parameters from measured data and its ability of monitoring and adapting online to their time evolution in real-world propagation conditions. We finally report on the performance verification results in Section 5 before we draw out our conclusions in Section 6.

\section{ANALYSIS/SYNTHESIS-BASED DATA MODEL AND RECEIVER DESIGN}

\subsection{Analysis/synthesis-based data model and assumptions}

For the sake of simplicity, we consider the uplink direction of a cellular CDMA system from a portable to a base station. ${ }^{1}$ Below we explain the data model and the STAR receiver structure in the general framework where we assume that the base station is equipped with $M$ receiving antennas. This is to underline the potential spatial-processing dimension of both the data model and the receiver. However, later we will illustrate the performance results with a database of real-world impulse response (IR) measurements recorded with a single receive antenna. Yet, as explained below, this database will largely serve the purpose of verifying ${ }^{2}$ the design and performance of STAR, the main scope of this work. We further consider a selective fading multipath environment characterized by $P$ propagation paths where the time delay spread $\Delta \tau$ is assumed small ${ }^{3}$ compared to $T$. The user's BPSK symbol sequence is first differentially $y^{4}$ encoded at rate $1 / T$, where $T$ is the symbol duration. The resulting sequence $b(t)$ is then spread with a long bipolar pseudorandom noise (PN) sequence $c(t)$ at a rate $1 / T_{c}$ where $T_{c}$ is the chip pulse duration. The spreading factor is given by $L=T / T_{c}$.

We denote the observation vector received by the antenna array as $X(t)$. A preprocessing unit in STAR passes $X(t)$ through a matched-pulse filter ${ }^{5}$ and provides the matchedfiltering observation vector for frame number $n$ over the time interval $\left[0,2 T-T_{c}\right)$ in successive overlapping frames of pe$\operatorname{riod} T$ by $[2]$

$$
Y_{n}(t)=\frac{1}{T_{c}} \int_{D_{\phi}} X\left(\frac{\delta_{T} T}{2}+n T+t+t^{\prime}\right) \phi\left(t^{\prime}\right) d t^{\prime}
$$

where $D_{\phi}$ denotes the temporal support of a truncated square-root raised-cosine shaping pulse $\phi(t)$ and $\delta_{T} \in\{0,1\}$ allows for a possible time shift by $T / 2$ to avoid locating the frame edges in the middle of the delay spread [1]. For simplicity, we assume $\delta_{T}=0$. After sampling at the chip rate and framing over $2 L-1$ chip samples at the symbol rate by the preprocessing unit, we obtain the $M \times(2 L-1)$ matchedfiltering observation matrix

$$
\mathbf{Y}_{n}=\left[Y_{n}(0), Y_{n}\left(T_{c}\right), \ldots, Y_{n}\left((2 L-2) T_{c}\right)\right]
$$

The structure of this matrix is detailed in [2]. After despreading $\mathbf{Y}_{n}$ rowwise:

$$
Z_{n}\left(l T_{c}\right)=\frac{1}{L} \sum_{k=0}^{L-1} Y_{n}\left((l+k) T_{c}\right) c\left(n T+k T_{c}\right)
$$

\footnotetext{
${ }^{1}$ Extension to downlink is similar irrespective whether the portable terminals are equipped with one or more receive antennas [8].

${ }^{2}$ Indeed, the analysis/synthesis approach of STAR applies identically to both time and space diversity extraction.

${ }^{3}$ Extension to delay spreads larger than the symbol duration for highspeed transmissions was addressed in [12].

${ }^{4}$ For simplicity, we considered the blind version of STAR. Efficient use of pilots in STAR was addressed in $[3,12]$.

${ }^{5}$ Please note that the model described is baseband without loss of generality [2].
} 
and framing the resulting post-correlation vector $Z\left(l T_{c}\right)$ over $L$ chip samples at the symbol rate:

$$
\mathbf{Z}_{n}=\left[Z_{n}(0), Z_{n}\left(T_{c}\right), \ldots, Z_{n}\left((L-1) T_{c}\right)\right]
$$

we obtain the $M \times L$-dimensional post-correlation observation matrix $\mathbf{Z}_{n}[1]$ :

$$
\mathbf{Z}_{n}=s_{n} \underbrace{\mathbf{H}_{n}}_{\text {ST channel }}+\mathbf{N}_{n}=s_{n} \underbrace{\left(\mathbf{J}_{n} \mathbf{D}_{n}^{T}\right)}_{\text {ST analysis }}+\mathbf{N}_{n}=s_{n} \underbrace{\left(\mathbf{G}_{n} \mathbf{\Upsilon}_{n} \mathbf{D}_{n}^{T}\right)}_{\text {ST analysis }}+\mathbf{N}_{n},
$$

where $s_{n}=b_{n} \psi_{n}$ denotes the signal component, $b_{n}=b(n T)$ is the transmitted DBPSK symbol, and $\psi_{n}^{2}$ is the total received power affected by the Doppler spread and multipath fading, the path-loss and shadowing and power control (PC). $\mathbf{H}_{n}$ is the $M \times L$ spatio-temporal channel matrix. $\mathbf{N}_{n}$ is the $M \times L$ spatio-temporal uncorrelated noise ${ }^{6}$ matrix after despreading with variance $\sigma_{N}^{2}$. It includes the thermal noise received at the antenna elements as well as the self-, in-cell and out-cell interference. We hence define the input SNR after despreading as SNR $=\bar{\psi}^{2} / \sigma_{N}^{2}$, where $\bar{\psi}^{2}$ denotes the average received power.

The most important feature in the equation above, referred to as the post-correlation model (PCM) in [1], is the spatial-temporal (ST) "analysis" of the channel matrix $\mathbf{H}_{n}$ by its parametric decomposition, ${ }^{7}$ under structural constraints, as the product of an $M \times P$ spatial channel matrix $\mathbf{J}_{n}$ and a $P \times L$ temporal channel matrix $\mathbf{D}_{n}^{T}$. Indeed, $\mathbf{J}_{n}=\mathbf{G}_{n} \mathbf{Y}_{n}$ is itself the product of $\mathbf{G}_{n}=\left[G_{1, n}, \ldots, G_{P, n}\right]$, the $M \times P$ columnwise normalized spatial channel matrix, and $\mathbf{Y}_{n}=$ $\operatorname{diag}\left[\varepsilon_{1, n}, \ldots, \varepsilon_{P, n}\right]$, the $P \times P$ diagonal matrix of normalized power ratios over multipaths $\varepsilon_{p, n}^{2}$ (i.e., $\sum_{p=1}^{P} \varepsilon_{p, n}^{2}=1$ ). More importantly, each column of $\mathbf{D}_{n}=\left[D_{1, n}, \ldots, D_{P, n}\right]$ belongs to a temporal manifold, ${ }^{8}$ that is, its vector elements are known functions of a given parameter, namely, the corresponding time delay $\tau_{p, n}$ of the $p$ th multipath:

$$
\begin{aligned}
D_{p, n} & =\mathcal{F}_{T}\left(\tau_{p, n}\right) \\
& =\left[\rho_{c}\left(-\tau_{p, n}\right), \rho_{c}\left(T_{c}-\tau_{p, n}\right), \ldots, \rho_{c}\left((L-1) T_{c}-\tau_{p, n}\right)\right]^{T},
\end{aligned}
$$

where $\rho_{c}(t)$ is a truncated raised-cosine pulse which corresponds to the correlation function of the square-root raisedcosine shaping pulse $\phi(t)$.

\footnotetext{
${ }^{6}$ Extension to the correlated-noise case is addressed in the multiuser detector version of STAR in $[4,8]$. We will briefly verify its performance over channel measurements later in Section 5.3.

${ }^{7}$ The new interference subspace rejection (ISR) paradigm proposed recently $[4,8]$ exploits similar parametric decompositions of the noise matrix $\mathbf{N}_{n}$.

${ }^{8}$ Similarly, it is possible to force the columns of $\mathbf{G}_{n}$ to belong to a spatial manifold, that is, $G_{p, n}=\mathcal{F}_{S}\left(\theta_{p, n}\right)$ is a known function of the direction of arrival of the $p$ th multipath [13] (e.g., in the ideal case of plane-wave propagation).
}

We explain below how the STAR receiver is designed to exploit this spatio-temporal channel analysis feature by "synthesis" of significantly improved spatio-temporal channel estimates that fit into the analysis structure above.

\subsection{Overview of analysis/synthesis-based design of STAR}

The core idea of STAR is the following: A main channel estimation module, referred to as decision feedback identification $(\mathrm{DFI})^{9}$ in [1], provides a coarse unconstrained estimate $\widetilde{\mathbf{H}}_{n}$ of the spatio-temporal channel. In an analysis-step, a space-time separation or decomposition of the channel followed by successive extraction of the temporal channel matrix $\hat{\mathbf{D}}_{n}$ (i.e., synchronization) $)^{10}$ and the spatial ${ }^{11}$ channel matrix $\widehat{\mathbf{J}}_{n}$ from $\widetilde{\mathbf{H}}_{n}$. In a synthesis step, a space-time reconstruction of the channel then provides a far more accurate constrained estimate [1]:

$$
\hat{\mathbf{H}}_{n}=\underbrace{\hat{\mathbf{J}}_{n} \hat{\mathbf{D}}_{n}^{T}=\hat{\mathbf{G}}_{n} \hat{\mathbf{Y}}_{n} \hat{\mathbf{D}}_{n}^{T}=\hat{\mathbf{G}}_{n} \hat{\mathbf{Y}}_{n}\left[\mathcal{F}_{T}\left(\hat{\tau}_{1, n}\right), \ldots, \mathcal{F}_{T}\left(\hat{\tau}_{\hat{P}, n}\right)\right]^{T}}_{\text {ST channel synthesis }},
$$

by structure fitting along the nominal decomposition of the channel in (5) and (6) of the PCM model. A final combining step exploits the constrained channel estimate $\hat{\mathbf{H}}_{n}$ to extract the signal component ${ }^{12} \hat{s}_{n}$ using simple MRC [2] or interference suppression [4]. Mathematical details can be found in $[2,4]$. For simplicity here, we explain the concept and advantages of the analysis/synthesis-based design of STAR following the illustrations in Figure 1.

The power of the spatio-temporal channel $\mathbf{H}_{n}=$ $\left[H_{n, 1}, \ldots, H_{n, k}, \ldots, H_{n, L}\right]$ is depicted in Figure 1a. It suggests that seven coefficients $H_{n, k}$, at least, have nonnegligible power. In Figure 1b, the power of the despread observation $\mathbf{Z}_{n}$ of (4) shows that these seven most desired channel components are buried in noise. Conventional correlator-type receivers would average unmodulated post-correlation observations over time to bring these useful channel coefficients above a detection threshold. In contrast, the DFI module in STAR identifies the channel blindly using simple adaptive subspace tracking $[1,3]$. As shown in Figure 1c, the resulting unconstrained estimate $\widetilde{\mathbf{H}}_{n}=\left[\widetilde{H}_{n, 1}, \ldots, \widetilde{H}_{n, k}, \ldots, \widetilde{H}_{n, L}\right]$ constantly keeps the power of most of the desired channel components above the rest of the coefficients (which may sporadically exceed the detection threshold unnoticed [2]). Yet, it offers only a coarse estimate of the actual channel coefficients in Figure 1a.

\footnotetext{
${ }^{9} \mathrm{DFI}$ is an adaptive subspace tracking technique that implements "quasicoherent blind" or "quasiblind coherent" detection [3].

${ }^{10}$ The columns $\hat{D}_{p, n}$ are reconstructed using (6) after estimation of the multipath time delays $\hat{\tau}_{p, n}$ and their number $\hat{P}$.

${ }^{11} \widehat{\mathbf{G}}_{n}$ and $\hat{\mathbf{Y}}_{n}$ are simply estimated by columnwise normalization of $\hat{\mathbf{J}}_{n}$.

${ }^{12}$ The received power estimate $\hat{\psi}_{n}^{2}$ and the symbol estimate $\hat{b}_{n}$ are extracted from $\hat{s}_{n}$ by power smoothing and hard decision, respectively.
} 


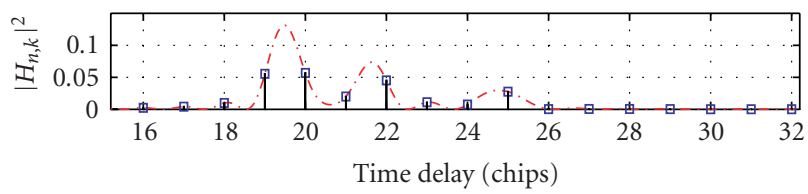

(a)

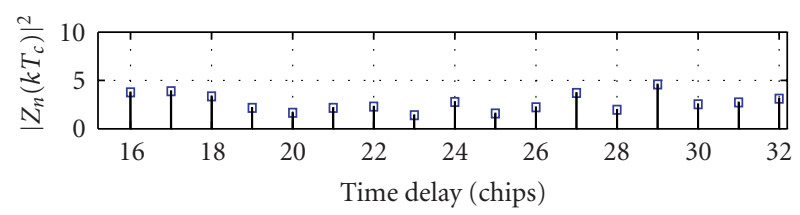

(b)

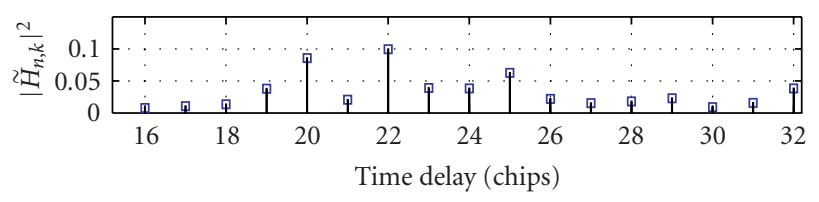

(c)

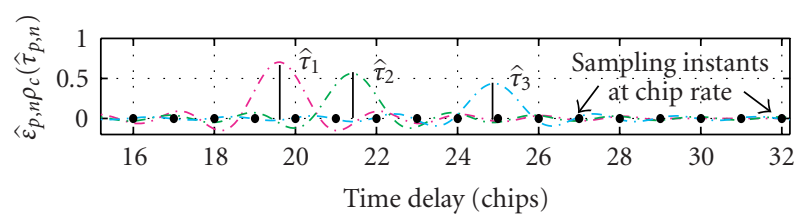

(d)

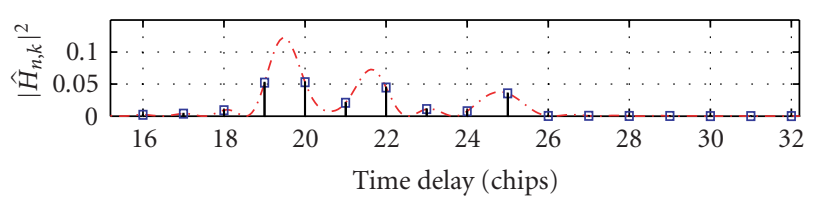

(e)

FIGURE 1: Channel analysis/synthesis in STAR illustrated with $M=1$ receive antenna: (a) power of chip rate channel coefficients (power waveform is in dashed line), (b) power of chip-rate despread observation, (c) power of unconstrained estimate of chip-rate channel coefficients, (d) channel analysis by high-resolution extraction from $\widetilde{\mathbf{H}}_{n}$ of time delays $\hat{\tau}_{p, n}$ and their number $\hat{P}=3$, and (e) power of constrained estimate of chip-rate channel coefficients after synthesis (resulting power waveform is in dashed line) by summation of $\hat{P}=3$ replicas of a truncated raised-cosine pulse, each delayed by $\hat{\tau}_{p, n}$ and weighted by $\widehat{J}_{p, n}=\widehat{\varepsilon}_{p, n} \hat{G}_{p, n}$, respectively, followed by chip-rate sampling.

It is at this stage that the analysis/synthesis design features contribute significantly to the enhanced performance of STAR. Exploiting the parametric decomposition of the channel in (5) and (6), STAR extracts by a high-resolution technique [1] the multipath time delays $\widehat{\tau}_{p, n}$ and their number $\hat{P}$ from the unconstrained channel estimate $\widetilde{\mathbf{H}}_{n}$ depicted in Figure 1c. In Figure 1d, the multipath time delays are accurately located with a resolution unlimited by the sampling rate or the clock precision. In contrast, RAKEtype receivers require oversampling above the chip rate or clock shifts by chip fractions [2]. Estimation of the normalized multipath amplitudes $\widehat{\varepsilon}_{p, n}$ and the complex fading components $\widehat{G}_{p, n}$ completes the analysis step and allows for reconstruction of the constrained channel estimate $\hat{\mathbf{H}}_{n}=$ $\left[\widehat{H}_{n, 1}, \ldots, \hat{H}_{n, k}, \ldots, \hat{H}_{n, L}\right]$ in the synthesis step. In Figure 1e, the resulting enhanced channel estimate is the best constrained fit that can be extracted from the unconstrained estimate $\widetilde{\mathbf{H}}_{n}$ depicted in Figure 1c. It exploits all desired channel coefficients, more than seven in number, by constantly monitoring three parameters only, ${ }^{13}$ namely the multipath

\footnotetext{
${ }^{13}$ Accordingly, channel analysis and synthesis could be viewed as coding and decoding in quantization.
} 
time delays. In contrast, RAKE-type receivers monitor a preselected number (around 5 fingers) of despread data elements in $\mathbf{Z}_{n}$ (see Figure $1 \mathrm{~b}$ ) at variable sampling instants below the chip rate, using an independent early-late gate (ELG) for each correlation branch. Advantages of STAR over RAKE-type receivers $^{14}$ are well documented in [2]. In the next section, we explain our approach to validating the analysis/synthesisbased design and performance of STAR using real-world measurements, the scope of this work.

\section{DESIGN AND PERFORMANCE VERIFICATION APPROACH}

Our verification work aims to provide two main sets of results: (1) assess the ability of STAR to carry out online extraction of the channel parameters from real-world measurements to verify its design, (2) assess the mismatch between the simulator results (at both link and system levels) obtained with generic models fed with the extracted parameters and those obtained with the channel measurements. In the next subsections, we explain the methodology adopted to get these results then provide brief descriptions of the real-world measurements and the simulator setup.

\subsection{Methodology}

In order to verify both the design and performance of the analysis/synthesis-based receiver STAR, we divided our approach into three highly interconnected steps (as illustrated in Figure 2):

(1) dynamic channel characterization of real-world wideband radio channels,

(2) further development, upgrade, and optimization of the STAR receiver,

(3) realistic system-level performance verification of the STAR receiver.

Indeed, successful dynamic extraction of real-world radio-channel parameters under step (1) above allowed for the verification of the analysis/synthesis-based design of STAR and pointed out places for improvement of this adaptive receiver under step (2) both in performance and complexity. In turn, enhancements in the STAR receiver under step (2) increased its dynamic channel parameter extraction capabilities and therefore improved the dynamic characterization of wideband radio channels under step (1). This twoway link-level development approach based on comparisons between the generic models of the wideband radio channel parameters exploited by the STAR receiver and their extracted real-world realizations was reassessed from a systemlevel point of view under step (3). This step raised issues of its own related to generic/real-world channelmismatch analysis

\footnotetext{
${ }^{14}$ Unfortunately the database, sampled only at half the chip rate (cf. Section 3.2), does not permit a reliable performance verification of the earlylate gate (ELG) or digital delay-locked loop (DDLL) component of RAKEtype receivers with a fair time resolution of at least $T_{\mathcal{c}} / 4$.
}

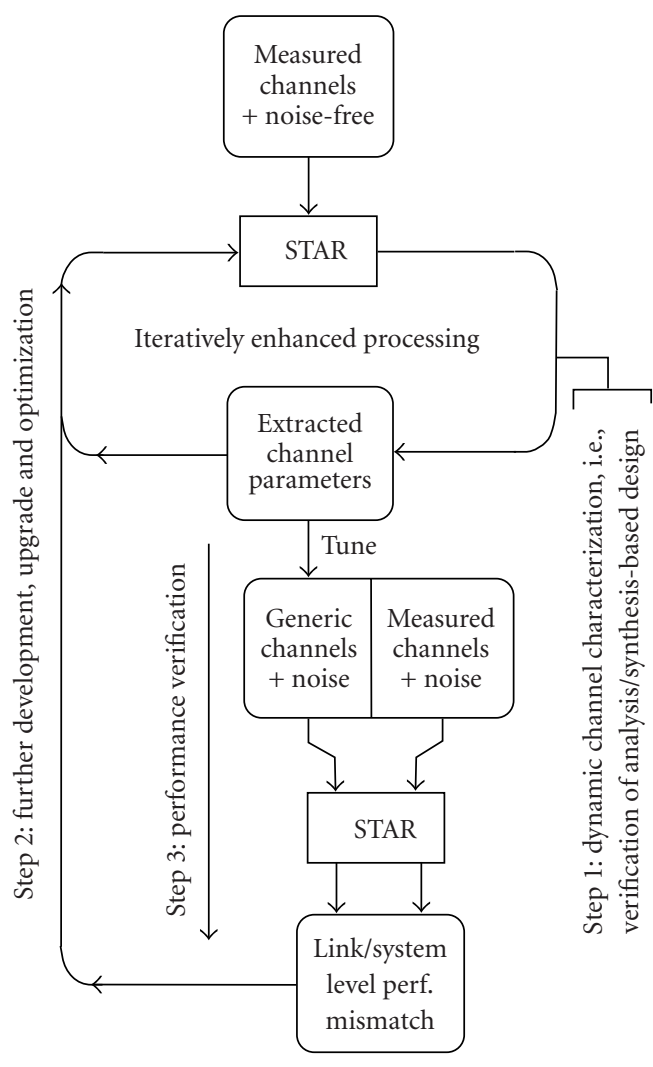

FIGURE 2: Block diagram of the design verification and performance evaluation methodology.

and enhanced modeling which led to improved dynamic characterization of wideband radio channels under step (1). Under step (3), we were also able to develop a faster and more reliable system-level software simulator. Based on the performance assessment made there in a more realistic simulation environment, we have been able to issue recommendations on privileged directions for further development, upgrade, and optimization of STAR under step (2).

Clearly, the three steps above were highly interactive and required an iterative approach (see Figure 2). For the sake of clarity, we skip the details ${ }^{15}$ and rather focus on the results of this approach. In the next section, we will illustrate the on-line parameter extraction capability of STAR from realworld measurements to verify its analysis/synthesis-based design. Later we will compare both the link- and system-level results obtained with generic channel models (fed with the extracted parameters) with those obtained with the realworld channels to verify its performance. Besides achieving these design and performance verifications, the methodology adopted opened out onto a more appropriate and new dynamic characterization of wideband radio channels as well as

\footnotetext{
${ }^{15}$ However, by underlining in Section 4 the role each extracted channel parameter played in our adaptive receiver design, we will highlight the multiple dimensions for performance/design/complexity/modeling reoptimization and reassessment that we have addressed in this iterative process.
} 
an improved software simulator both in reliability and speed. Before we discuss these results in the following sections, we provide below brief descriptions of the real-world database and the software simulator.

\subsection{Real-world database}

A database of real-world radio-channel measurements was recently made available for this study by Microcell Solutions, a wireless service provider in Canada. The database contains, in a raw format, impulse response (IR) measurements of $5 \mathrm{MHz}$ radio-channels recorded between a roof-top transmit antenna ${ }^{16}$ and a single receive antenna (i.e., $M=1$ ) mounted inside a mobile minivan. The channel sounder, shown in Figure 3, was operated in the North American PCS band at a carrier frequency of $1982.5 \mathrm{MHz}$ by continuously transmitting an unmodulated $\mathrm{PN}$-sequence of 255 chips at the former WCDMA chip rate of $4.096 \mathrm{Mcps}$ (later fixed to 3.840 Mcps $[14,15])$. After capturing the off-air signal via a wideband monopole antenna, the received signal was successively passed through a preselector filter, a low noise amplifier (LNA), an image reject filter, followed by a single-conversion quadrature down-converter. The $I$ and $Q$ components were sampled at a rate of $8.192 \mathrm{MHz}$ (oversampling by a factor of 2) using a 12-bit-resolution A/D for each output. Note, however, that STAR does not require oversampling and will only exploit the measurements at the chip rate. Each quadrature signal was acquired every 1 msecond and was continuously streamed to the disk. Full details of the recording setup and conditions are provided in [16].

From this database, we have been able to exploit the very last set of four IR recordings conducted at the so-called site 132 in Laval (a suburban area of Montreal) using an upgraded version of the channel sounder [16]. Indeed, previous recordings suffered from very large and frequent hops in synchronization between successive IR acquisitions, which severely distort (if not destroy) the natural time evolution of the channel required in this work. In the initial 3G trial conducted in 1999, measurements were collected with the intention of carrying out a conventional statistical channel characterization [16] where even independent IR snapshots could have been exploited; thus use of a higher-cost channel sounder was not justified.

Despite the equipment upgrade, two of the IR recordings exploited in this work still contained large yet sporadic synchronization hops. Such imperfections are very unlikely in commercial wireless terminals and could have been removed manually by a simple temporal alignment between successive IR measurements. However, we decided not to do so in order to illustrate the robustness and the tracking capabilities of the STAR receiver. Preprocessing of the four retained recordings was only limited to removing the IR components below the noise floor of the channel sounder. The resulting IRs are illustrated in Figure 4. They correspond to four different routes of the minivan close to a mall in the suburban Laval area (i.e., site 132). Documentation specifies only a maximum

\footnotetext{
${ }^{16}$ Fixed at various Microcell's urban or suburban antenna cell sites.
}

speed of $30 \mathrm{~km} / \mathrm{h}$ in all recordings [16]. The first three recordings have a 170-second duration each while the fourth is 80 seconds (i.e., around 600000 IR measurements in total), all long enough to allow for reliable design and performance verifications.

\subsection{Simulator setup}

The simulator is composed of a link-level module and a system-level one. The link-level module integrates STAR in a transceiver structure that includes a channel coder, an interleaver, a spreader, and a power amplifier at the transmitter, a baseband multipath Rayleigh-fading ${ }^{17}$ channel generator that adds white Gaussian noise to simulate all sources of interference, a despreader, the STAR receiver, a deinterleaver, a channel decoder, and a power control (PC) unit at the receiver. For a specified input SNR level, it provides link-level statistics such as the symbol error rate (SER) or frame error rate (FER) as well as transmit and receive power distributions. The system-level module uniformly populates a square grid of cells with a total number of users and measures the total received interference at the base station of the central cell taking into account the transmit and receive power distributions. For a specified capacity $C$ defined as the average number of users per cell, it provides the outage probability that the signal-to-interference ratio is below a given input SNR. More details can be found in $[2,17]$.

The baseband channel generator was first modified to operate with both generic models and IR measurements directly fed to the link-level module. In the dynamic channel characterization or design verification phase (see Figure 2), only the link-level module is needed and the channel generator there is operated with the IR measurements, noise-free for the sole purpose of extracting the channel parameters. ${ }^{18}$ In the second performance verification phase (see Figure 2), both modules are first operated with generic channels tuned by the parameters extracted in the first phase, then with the measured channels.

As reported in Table 1 (unless specified otherwise), we ran the simulator at a carrier frequency of $1.9825 \mathrm{GHz}$ and a chip rate of $4.096 \mathrm{Mcps}$. User data at a voice rate of $8 \mathrm{kbps}$ was encoded using a rate- $1 / 2$ convolutional code with constraint length of 9 then spread with long PN spreading sequences with a spreading factor $L=256$. Despreading was limited to a reduced processing window around the delay spread of $L_{\bar{\Delta}}=32$ chips (see details in [2]). We used squareroot raised-cosine (RRC) shaping pulses ${ }^{19}$ with a rolloff of $0.22[14,15]$ truncated to 17 coefficients at the chip rate. Imperfect PC was simulated by transmitting power-change

\footnotetext{
${ }^{17}$ Study of the measurements did not reveal line-of-sight components typical of Ricean channels.

${ }^{18}$ Actually, running the module at operating SNR levels resulted in little mismatch with the parameter values extracted from noise-free observations. STAR can still extract the channel parameters online while receiving data over the wireless network (see footnote 20).

${ }^{19}$ We could not find the specifications of the pulse shape used by the sounder. We hence assumed the standards' specification.
} 


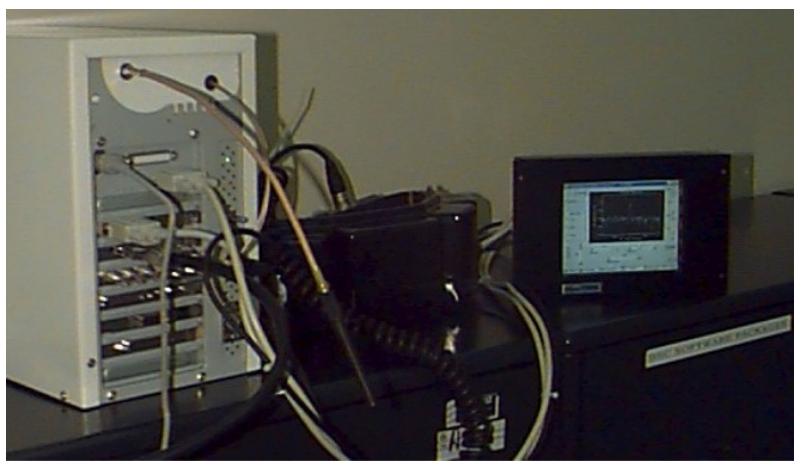

FIGURE 3: Photograph of the channel sounder.

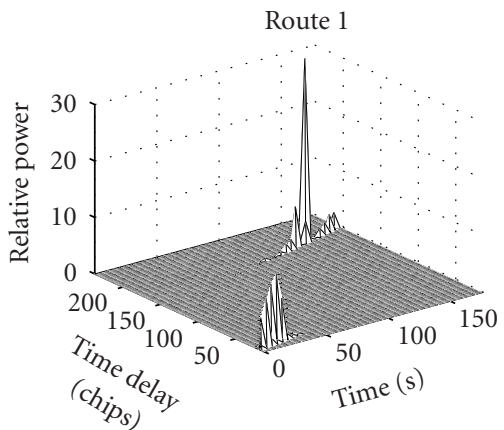

(a)

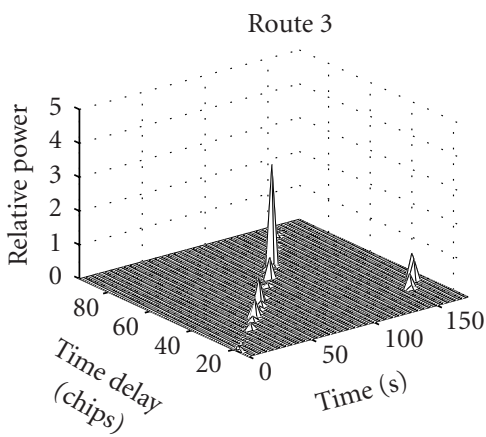

(c)

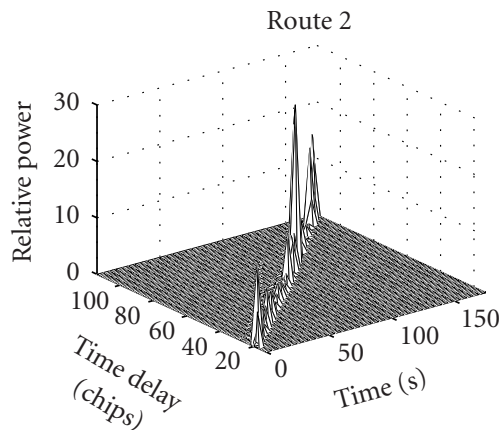

(b)

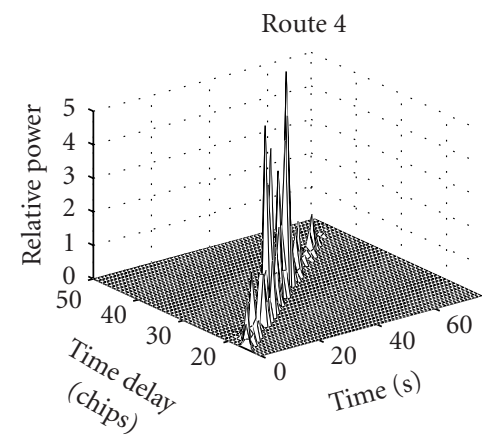

(d)

FIGURE 4: Power versus IR coefficient and time of the channel recordings collected along four different routes in Laval, a suburban area of Montreal, at vehicular speed below $30 \mathrm{~km} / \mathrm{h}$.

commands of $\pm 0.25 \mathrm{~dB}$ at a rate of $1600 \mathrm{~Hz}$ with transmission delay of 0.625 milliseconds and error rate of $5 \%$.

\section{RECEIVER DESIGN VERIFICATION RESULTS}

\subsection{Extraction of the multipath time delays}

Timing recovery is one of the most critical functions of a receiver [18]. Variation in time delays is caused by the mobility of the receiver (or transmitter) and the imprecision of internal clocks or local oscillators. It renders synchronization even more critical by requiring constant tracking and frequent updates of the time delays. We have shown with generic time-evolution models for the delays that these drifts have a serious impact on the performance of enhanced wideband CDMA array receivers $[1,2]$. Here, we extract the multipath time delays from the channel measurements to verify the analysis/synthesis-based design of STAR and to illustrate both its time synchronization capability and its ability to generate realistic time-evolution models for the multipath delays. 
TABle 1: Parameters used in the simulations (unless specified otherwise).

\begin{tabular}{lcl}
\hline Parameter & Value & Comment \\
\hline$f_{c}$ & $1.9825 \mathrm{GHz}$ & Carrier frequency \\
$R_{c}$ & $4.096 \mathrm{Mcps}$ & Chip rate \\
$R_{b}$ & $8 \mathrm{kbps}$ & Bit rate \\
$r$ & $1 / 2$ & Coding rate \\
$K$ & 9 & Constraint length \\
$M$ & 1 & Number of receive antennas \\
$L$ & 256 & Spreading factor \\
$L_{\bar{\Delta}}=32$ & 32 chips & Reduced processing window \\
$\beta$ & 0.22 & RRC rolloff factor \\
$N_{c}$ & 17 & Number of RRC coefficients \\
$f_{\mathrm{PC}}$ & $1600 \mathrm{~Hz}$ & Frequency of PC updating \\
$\Delta P_{\mathrm{PC}}$ & $\pm 0.25 \mathrm{~dB}$ & PC adjustment \\
$\tau_{\mathrm{PC}}$ & 0.625 milliseconds & PC feedback delay \\
BER & $5 \%$ & Simulated PC bit error rate \\
\hline
\end{tabular}

In Figure 5, we plot both the power contour of the four IR recordings as well as the corresponding multipath time delays extracted by STAR. Bearing in mind the illustration previously made in Figure 1 of the analysis/synthesis feature of STAR (cf. Section 2.2), we readily verify the validity of its concept over real-world measurements in Figure 5 by relating, respectively, the power waveform (in dashed line) in Figure 1a and the train of pulses at the multipath locations in Figure 1d to two vertical cross-sections of Figures $5 \mathrm{a}$ and $5 \mathrm{~b}$ taken at the same time index. Indeed, most of the useful IR components are properly captured from the real-world channel measurements.

In Table 2, we provide for each route the time percentage $^{20} P_{t}$ of tracking each detected path out of the total recording duration. In all four recordings, we find that the channel is characterized by a maximum number of three multipaths. The first two paths, the strongest as discussed in the next section (see Table 2 ), are basically extracted $100 \%$ of the time (one to two chips apart from each other on average). Depending on the route, the weakest path is detected and tracked (one to few chips away from the others) from about 50 to $80 \%$ of the time, still a very good performance in time delay extraction (considering the power levels in Table 2). Additionally, the time delays extracted for routes 1 and 3 further demonstrate the very fast tracking capabilities of STAR by instantly responding to very large time delay hops. Although such imperfections were unlikely in commercial wireless terminals (cf. Section 3.2), they required reoptimization of synchronization that was useful in solving another challenge, much more practical. Indeed, by automatically positioning the edges of the reduced-size processing window [2] around the delay spread whenever a time delay

\footnotetext{
${ }^{20}$ To illustrate the parameter extraction capacity of STAR, we provide values for $P_{t}$ found for a practical input SNR of $5 \mathrm{~dB}$ (see Section 5 and Table 3 ). The same range of values for $P_{t}$ is obtained with simulated channels [19].
}

hop occurred, the reoptimized synchronization module implicitly addressed the previously simple yet open question of keeping the edges of the post-correlation observation frame outside the delay spread (see comment on parameter $\delta_{T}$ below (1)).

Besides allowing timely extraction of the channel parameters, analysis/synthesis-based STAR enables online dynamic modeling of their time-evolution from real-world realizations. The time delay profiles extracted in Figure $5 \mathrm{~b}$ are only examples that clearly illustrate their much broader dynamic characterization capability. They particularly suggest for the four channel recordings considered that time delays could evolve linearly ${ }^{21}$ in time in the long term, with a delay-drift or slope $\widehat{d \tau / d} t$ in Table 2 specifically ranging here ${ }^{22}$ between 0.10 and $0.14 \mathrm{ppm}$ (particle per million). A closer look at the time variations suggest slightly different drifts between paths for route 2 and shorter-term sinusoidal oscillations around the linear drift for route 4 . These results suggest that processing of more data could allow perfection of previously suggested models $[14,20]$ where time evolution of multipath delays was assumed sinusoidal. ${ }^{23}$

\subsection{Extraction of the multipath components}

Obviously, extraction of the multipath fading channel components is essential for coherent combining, interference suppression, decoding, and so forth. Here, we are particularly interested in the dynamic characterization of their time evolution. Time variations of multipath amplitudes may have an impact on the acquisition and tracking capabilities of a synchronization module and consequently on the performance of enhanced CDMA array receivers. They may impose stringent efforts to tune multipath detection thresholds above the appropriate fading levels $[2,19]$. With generic models, we have been particularly able to show the robustness of STAR to significant changes in the average multipath power profile [19]. Successful extraction of the dynamic multipath fading profiles from the channel measurements allows verification of the analysis/synthesis-based design of STAR and enables online monitoring of the average multipath power profile for automatic receiver-setup optimization in continuously changing environments.

In Figure 6a, we plot the dynamic power profile of the first multipath component as extracted ${ }^{24}$ by STAR. The other

\footnotetext{
${ }^{21}$ In the general case, evolution profiles will depend on the user mobility and clock or oscillator imperfections.

${ }^{22}$ The measured drifts are higher than the maximum clock imprecision thresholds of 0.05 and $0.1 \mathrm{ppm}$ allowed by recent $3 \mathrm{G}$ standard proposals on the uplink and the downlink, respectively $[14,15]$.

${ }^{23}$ Ideally, the selected benchmark profile should reflect a realistic histogram of a maximum number of measured drift values for a given environment.

${ }^{24}$ We plot $\widehat{\psi}_{n}^{2}\left|\widehat{J}_{1, n}\right|^{2} / \hat{a}_{n}^{2}$, where $\hat{a}_{n}^{2}$ is the transmit power profile of the mobile station reconstructed at the base station from the transmitted PC instructions. Note that STAR would require $\hat{a}_{n}^{2}$ only to characterize the largescale variations. Furthermore, analysis of the measurements revealed a weak high-frequency multiplicative noise. We hence introduced lowpass filtering of $\widehat{\boldsymbol{J}}_{n}$ elements.
} 

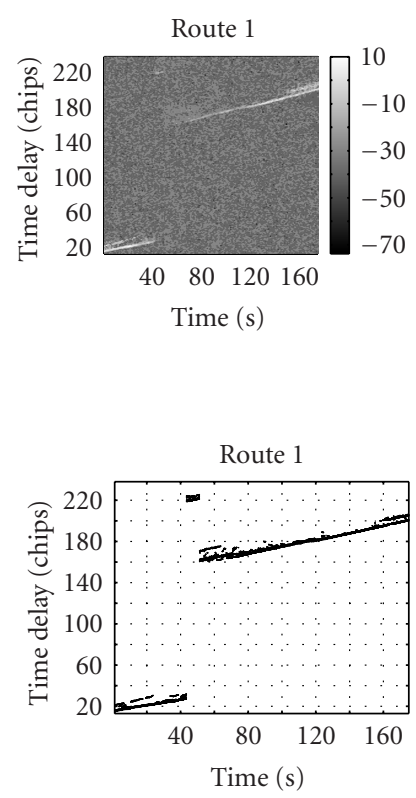
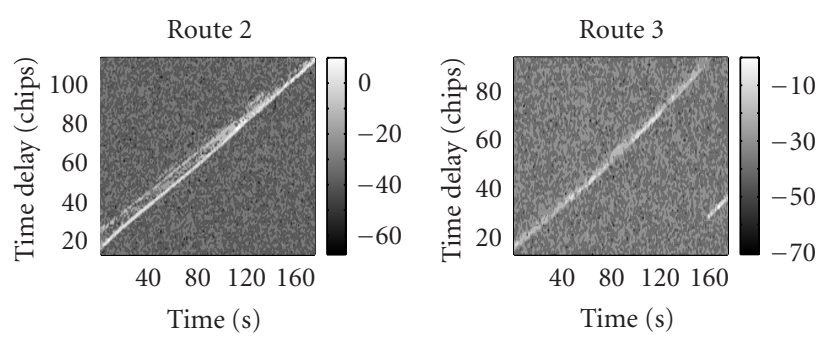

(a)

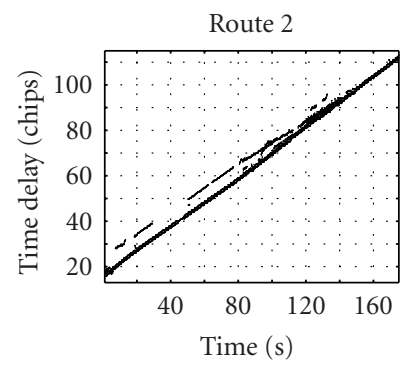

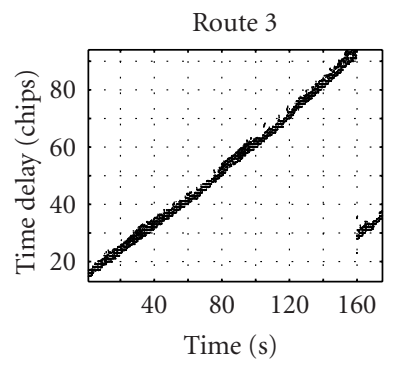

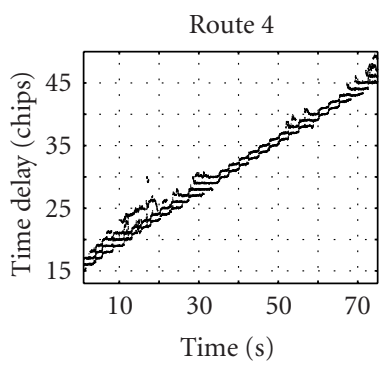

(b)

FIGURE 5: Extraction of time delays illustrated: (a) power contour of the four IR recordings in Figure 4 and (b) corresponding multipath time delays extracted.

TABle 2: Multipath extraction percentage over time $P_{t}$ and extracted channel parameters from data (delay drift $\widehat{d \tau / d} t$, average multipath power profile $\bar{\varepsilon}_{p}^{2} / \bar{\varepsilon}_{1}^{2}$, carrier frequency offset $\bar{\Delta} f$, maximum Doppler spread $\hat{f}_{D}$, or speed $\left.\hat{v}_{\max }\right)$.

\begin{tabular}{|c|c|c|c|c|c|}
\hline \multicolumn{2}{|c|}{ Parameter } & Route 1 & Route 2 & Route 3 & Route 4 \\
\hline \multirow{3}{*}{$P_{t}[\%]$} & 1st path & 100 & 100 & 100 & 100 \\
\hline & 2nd path & 99 & 97 & 99 & 99 \\
\hline & 3rd path & 71 & 60 & 85 & 48 \\
\hline$\widehat{d \tau / d} t(\mathrm{ppm})$ & - & 0.10 & 0.14 & 0.12 & 0.10 \\
\hline \multirow{3}{*}{$\left(\bar{\varepsilon}_{p}^{2} / \bar{\varepsilon}_{1}^{2}\right)(\mathrm{dB})$} & 1st path & 0 & 0 & 0 & 0 \\
\hline & 2nd path & -3.9 & -4.3 & -3.8 & -4.3 \\
\hline & 3rd path & -7.8 & -8.0 & -7.7 & -7.7 \\
\hline $\bar{\Delta} f(\mathrm{~Hz})$ & - & 179 & 61 & 89 & 120 \\
\hline$\widehat{f_{D}}(\mathrm{~Hz})$ & - & 42 & 30 & 41 & 10 \\
\hline$\hat{v}_{\max }(\mathrm{km} / \mathrm{h})$ & - & 23 & 17 & 22 & 6 \\
\hline
\end{tabular}

components, omitted for lack of space, show the same largescale fading variations. These can be approximated by longterm averaging or smoothing as illustrated in dashed line in Figure 6a. However, the three paths have quasi-uncorrelated small-scale fading variations, as illustrated in Figure $6 \mathrm{~b}$ between the first and second multipaths. Indeed, the correlation factor does not exceed 0.2 for all but the second route, where it is a little higher at around 0.35 . This result is very significant in that it strongly suggests that the analysis/synthesis-based receiver STAR is able to decompose the channel into few quasi-independent component ${ }^{25}$ using real-world data.

From the dynamic power profiles of the multipath components, STAR is also able to extract the corresponding average multipath power profile $\bar{\varepsilon}_{p}^{2} / \bar{\varepsilon}_{1}^{2}$ by simply taking $\bar{\varepsilon}_{p}^{2}$ as the mean over time of the normalized multipath power fraction estimate $\hat{\varepsilon}_{p, n}^{2}$. In Table 2 , we provide the average multipath power profile found for each route. As expected, the reported values suggest that the profiles are almost the same and hence typical of a single environment, namely, the suburban Laval area where the four measurements took place. With a typical profile of $[0,-4,-8] \mathrm{dB}$ similar to those specified by standards for the same suburban environments, we have been able to verify that the multipath detection module in STAR works nearly the same for both the generic and measured channels. For a path $4 \mathrm{~dB}$ weaker than the strongest path, tracking is barely lost, $1 \%$ of the time only (see Table 2). For a path $8 \mathrm{~dB}$ weaker than the strongest path, tracking is only lost about 20 to $50 \%$ of the time. More importantly, this result underlines the capacity of STAR of monitoring online the real-world environment where it operates and its ability to adjust to changes in the propagation conditions.

\subsection{Extraction of the carrier frequency offset}

Frequency synchronization is as crucial as timing in a wireless communication system [18]. Mismatch between the carrier frequencies at the transmitter and the receiver, that is,

\footnotetext{
${ }^{25}$ This feature, known in the literature as independent component analysis, is often implemented by subspace tracking, a technique used in the highresolution synchronization module of STAR [1].
} 

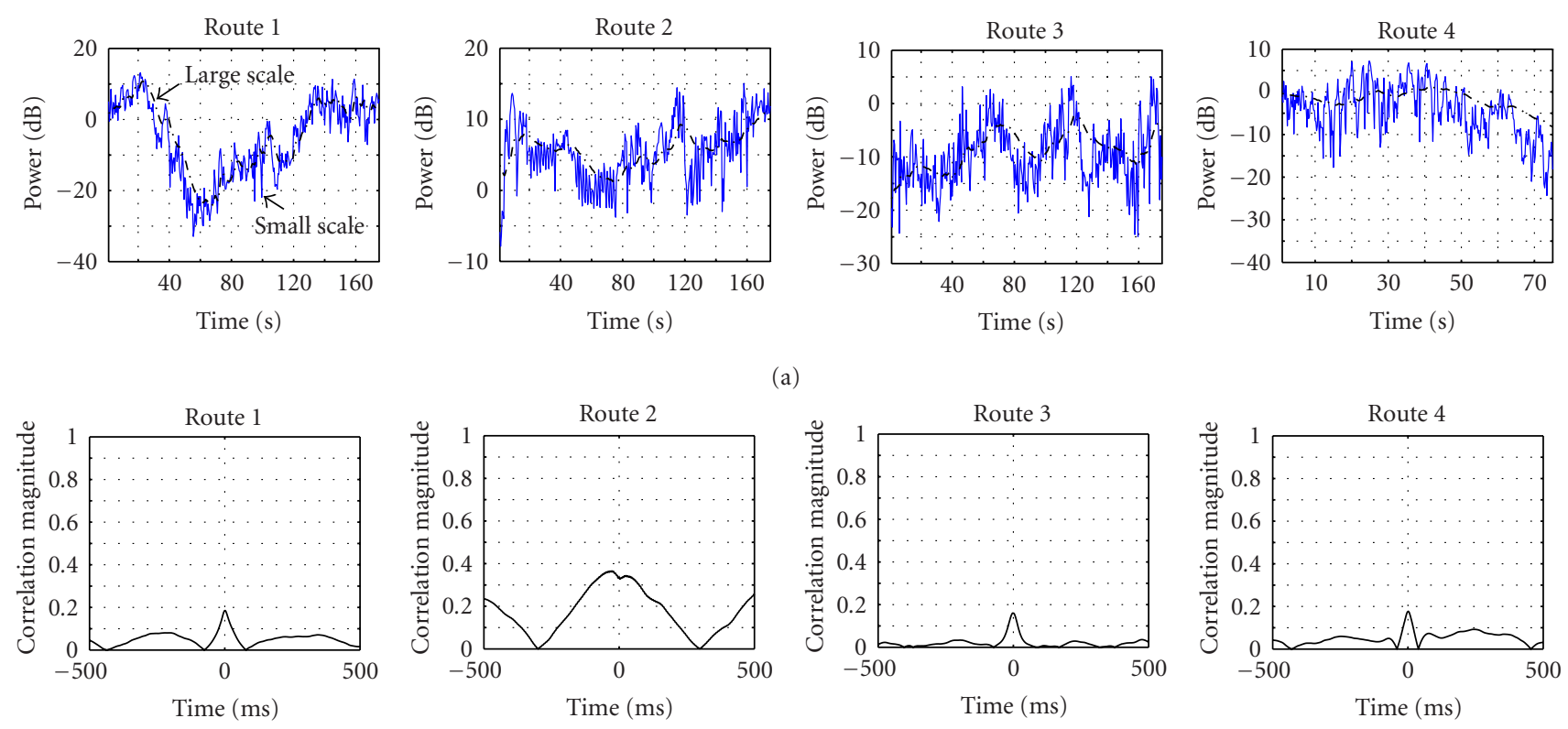

(b)

FIgURE 6: Extraction of multipath components illustrated: (a) power profile of the first multipath fading component and (b) magnitude of correlation factor between envelopes of first and second multipath components.

the carrier frequency offset (CFO), could have a serious impact on receiver performance, especially in coherent transmissions. We have shown in [5] that even CFO values below the tolerance thresholds of 0.10 and $0.05 \mathrm{ppm}$ specified by the standards on the downlink and uplink, respectively, could result in noticeable losses in SNR. We have ever since undertaken to integrate CFO recovery (CFOR) in STAR [5] as a further step along the same lines of its analysis/synthesisbased design. Indeed, assuming that a common CFO $\Delta f$ affects all multipath components (i.e., further analysis of spatial matrix as $\mathbf{J}_{n}=e^{-\Delta f} \mathbf{J}_{n}^{\prime}$, see (7)), STAR first estimates it by simple linear regression over the phase elements of $\widehat{\mathbf{J}}_{n}$ (details are not given in Section 2.2 for simplicity can be found ${ }^{26}$ in $[6,7])$ before matching it in the post-correlation observation (i.e., synthesis of new observation $\mathbf{Z}_{n}^{\prime}=e^{-\hat{\Delta} f} \mathbf{Z}_{n}$ ). This new CFOR module is able to estimate and compensate for CFOs and the resulting SNR losses efficiently, both in the single[7] and multiuser [6] receiver versions, using generic channel models. Here, we verify its analysis/synthesis-based design over real-world data by illustrating its ability to generate realistic time-evolution models for the CFO (currently unavailable to our knowledge).

In Figure $7 \mathrm{a}$, we plot as a reference the contour of the power spectral density ${ }^{27}$ (PSD) of the first multipath component extracted in Figure 6a without CFOR. In

\footnotetext{
${ }^{26} \mathrm{To}$ improve performance with measurements, note that we slightly modified the CFO update as $\widehat{\Delta} f_{n K}=\widehat{\Delta} f_{(n-1) K}+\alpha \hat{\delta} f_{n K}$, where $\hat{\delta} f_{n K}$ is an estimate of the adjustment residual and $\alpha$ is a smoothing factor.

${ }^{27}$ The PSD is calculated every 100 milliseconds by an FFT of the first multipath component autocorrelation over blocks of 512 milliseconds. Note here that we use $\hat{J}_{1, n}$ as extracted by STAR without compensation for the transmit power (see footnote 24 ).
}

Figure $7 \mathrm{~b}$, we plot the dynamic CFO profile extracted by STAR directly from the IR measurements. These figures clearly indicate that the CFOR module of STAR is able to track instantly and accurately the shifts of the Doppler spectrum from 0 due to CFO. This remarkable performance is better illustrated in Figure 7a where the average CFO $\bar{\Delta} f$ (from Figure $7 \mathrm{~b}$ ) perfectly matches with the shift from 0 of the average PSD ${ }^{28}$ (from Figure 7c). It confirms the capacity of STAR to eliminate the CFO and the resulting SNR losses from real-world measurements and opens out onto the possibility of modeling the CFO realizations. Furthermore, it enables estimation of the maximum Doppler spread (or speed) in the presence of CFOs as discussed in the next subsection.

The CFO profiles extracted in Figure $7 \mathrm{~b}$ have relatively weak and very slow time variations around the average CFO values $\bar{\Delta} f$ given in Table 2 . The recording durations are not long enough to build a reliable correlation profile from longterm statistics of the CFO process. They suggest, however, for short simulation times that the CFO could be fixed to a constant value randomly generated with a probability distribution that reflects real-world realizations. The cumulative histogram of the CFO realizations gathered from the extracted profiles is given in Figure 8 simply to illustrate such a possibility. More data is definitely needed.

\subsection{Extraction of the maximum Doppler spread or mobile speed}

Mobility degrades the performance of synchronization both in time and frequency. It also degrades channel identifica-

\footnotetext{
${ }^{28}$ It suggests the presence of a DC component and some harmonics due to filtering imperfections in the recording equipment.
} 

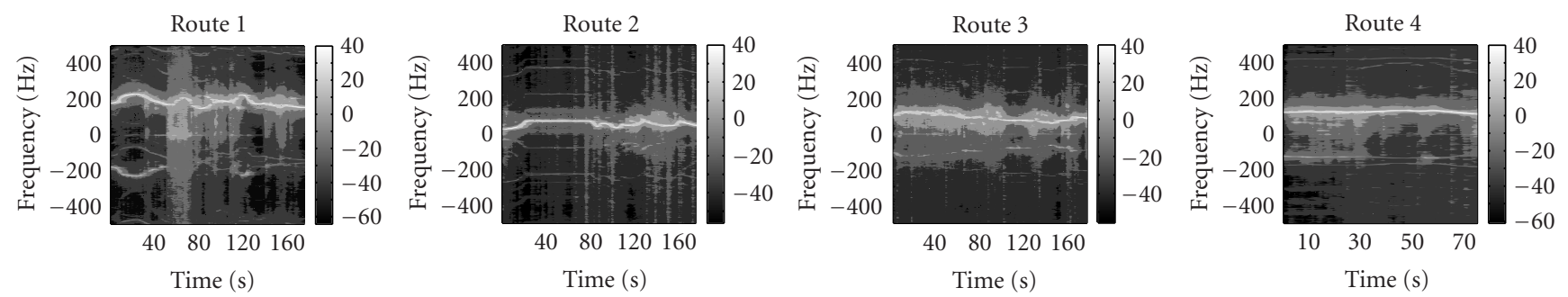

(a)
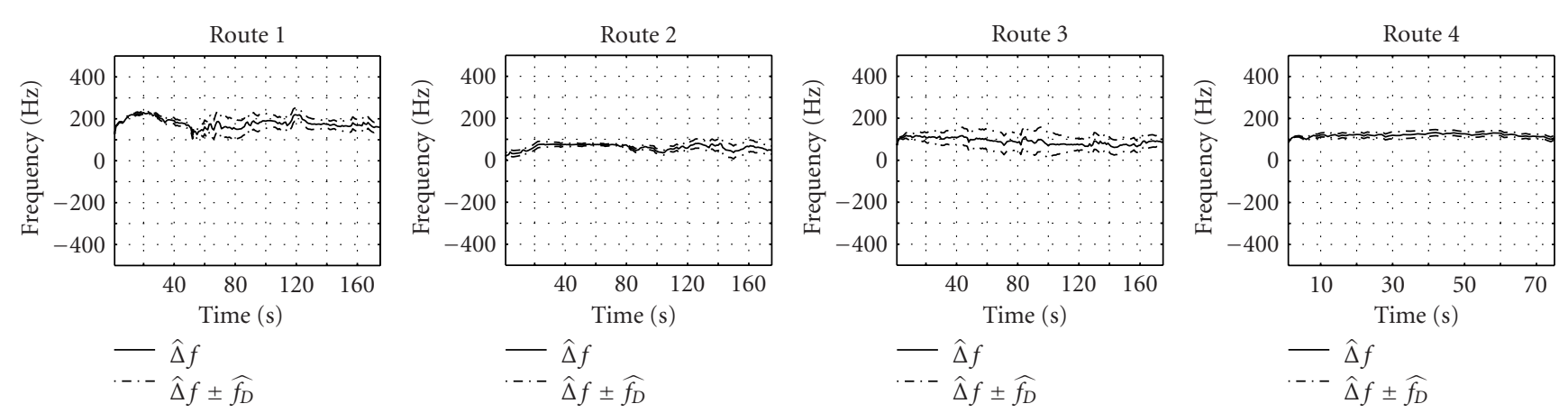

(b)
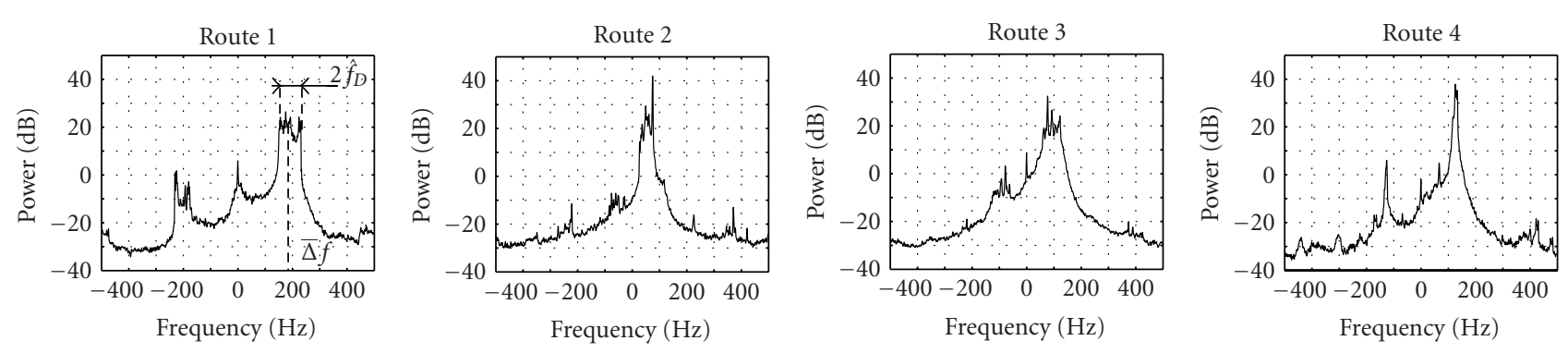

(c)
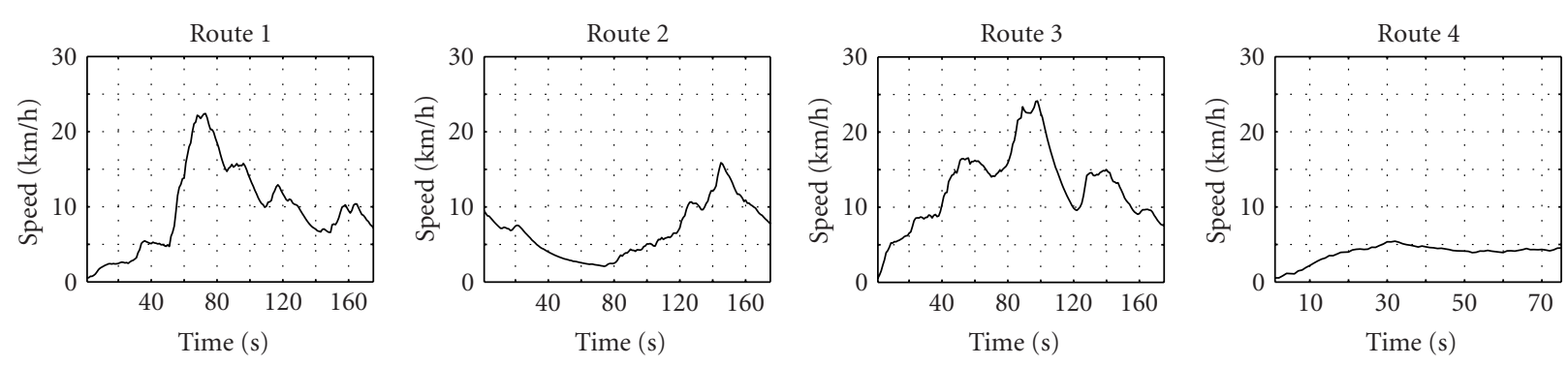

(d)

FIGURE 7: Extraction of the carrier frequency offset illustrated: (a) power spectral density contour of the first multipath component, (b) extracted carrier frequency offset (maximum Doppler spread around CFO is in dashed line), (c) average PSD of the first multipath component, and (d) estimated speed.

tion and tracking. In a thorough analytical study in [3], we found that the maximum Doppler shift $f_{D}$ or speed $v_{\max }$ is a key parameter in performance optimization of STAR and in the selection of its optimal adaptation step size. In another study, we have shown that computational resources can be allocated efficiently according to mobility (i.e., channel time variations) using a reduced channel-update rate option [2]. Below, we exploit the CFOR capabilities of STAR shown 


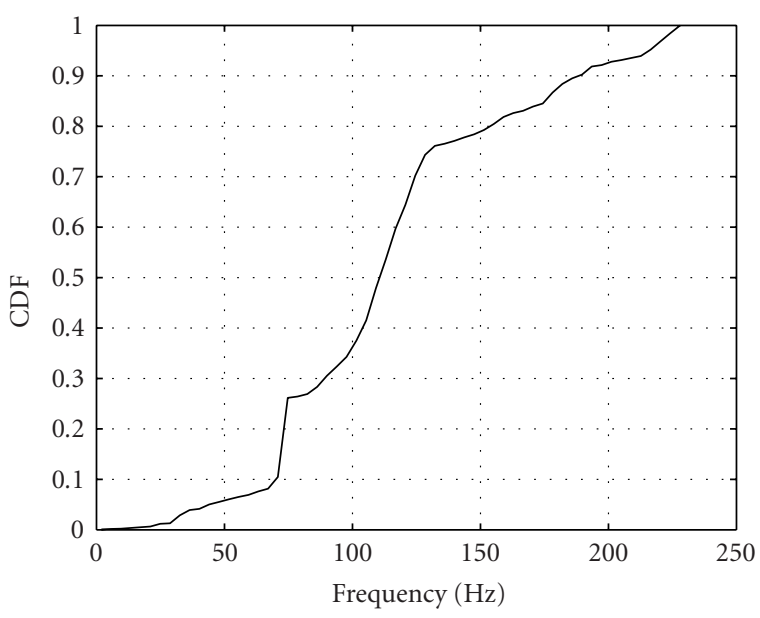

FIGURE 8: Cumulative histogram of CFO realizations (over equal durations of the CFO profiles in Figure $7 b$ ).

earlier to propose a simple technique for speed estimation in the presence of CFO and illustrate its performance using real-world measurements.

Previous Doppler estimators [21, 22, 23] assume a Ushaped Jakes' spectrum [24] centered on zero. In particular, the ML-based estimator in [22] proposes an efficient and simple implementation that amounts to maximizing over $f_{D}$ the scalar product between the PSD of the first multipath component (i.e., observation spectrum) and a Jakes' spectrum (i.e., nominal spectrum) with bandwidth $2 f_{D}$ centered at zero. Here, we modify the nominal spectrum, first, by centering it around the CFO estimate $\widehat{\Delta} f$, and second, by replacing its $U$ shape by a flat one. Indeed, the PSDs extracted from the measurements in Figure 6a did not have a Jake's spectrum (see average PSD in Figure 7c). Actually, the U-shaped Jakes' spectrum reflects only a particular 2D geometry of scatterers. In the absence of any a priori information on the scatterers' positions, we assume a $3 \mathrm{D}$ isotropically scattered field that results in a uniform spectrum [25].

With the above modifications, the ML-based Doppler estimator proposed in [22] reduces to a very simple procedure that automatically measures the mainlobe width $2 \hat{f}_{D}$ of the PSD around the CFO estimate $\widehat{\Delta} f$. As illustrated in Figure $7 \mathrm{c}$, the resulting technique provides in Table 2 the maximum Doppler and speed estimates from the average PSD over each recording duration. Using each PSD in Figure 7a, however, this technique can also provide continuous (or more frequent) estimation ${ }^{29}$ of the maximum Doppler as shown in dashed line in Figure $7 \mathrm{~b}$. The resulting speed estimates are given in Figure 7d. Although the estimated speed profiles appear realistic, the documentation available on the measurements does not allow us to corroborate them against the real

\footnotetext{
${ }^{29}$ An improved Doppler estimator in multipath fading could even be implemented by weighting the Doppler estimates from the different multipath components by the average power profile before their summation.
}

speed profiles. We can only verify that the true speed, indeed, never exceeded $30 \mathrm{~km} / \mathrm{h}$ [16].

Note that the speed extracted from the average PSD in Table 2 is about equal to the maximum value of the corresponding speed profile in Figure $7 d$, which suggests that the maximum speed is the critical parameter in the long term. Hence, for the performance verification below, we use generic channel models tuned with the constant maximum speed values reported in Table 2 (worst-case scenario).

\section{RECEIVER PERFORMANCE VERIFICATION RESULTS}

\subsection{Voice rate}

We have previously verified the analysis/synthesis-based design of STAR by illustrating its capability of extracting useful channel parameters, namely the multipath time delays, the multipath components, the CFO, and the maximum Doppler spread or speed. Here we verify its performance at both the link-level in terms of BER versus SNR, and at the system level in terms of maximum capacity (in users per cell) at $1 \%$ outage and $10^{-3} \mathrm{BER}$, by comparing the results obtained with measured channels and those obtained with the generic ones. As mentioned previously, we tune the generic channels with the parameter values ${ }^{30}$ reported in Tables 1 and 2 . Note, however, that we use the same average multipath power profile of $[0,-4,-8] \mathrm{dB}$ for simplicity. Furthermore, we set the initial multipath time delays to $[10,12,14] T_{c}$ before linear drift and set both the CFO and speed to be constant.

In Figure 9 and Table 3, respectively, we provide the linkand system-level performance results of STAR with both measured and generic channels. The BER curves in Figure 9 and the required SNR thresholds in Table 3 do not indicate any particular ordering in performance linked to the variations of a specific channel parameter in Table 2. More importantly, they show a constant gap of about 1.0 to $1.3 \mathrm{~dB}$ between the measured and generic channels resulting in 20 to $30 \%$ losses in capacity, regardless of the route. This is consistent with the fact that performance was optimized over all the design dimensions of STAR made available by analysis/synthesis of the channel, that is, in terms of timing (time delay parameter), CFOR (CFO parameter), and adaptive channel identification (multipath component and speed parameters). We attribute the mismatch of about $1 \mathrm{~dB}$ to other common sources of imperfection not taken into account in the generic channels. There are potentially many in practice: nonlinear multiplicative noise, shaping-filter distortions, adaptive gain control (AGC) errors, power amplifier saturation, and so forth, which all contribute to power leakage from the desired signal. There are very good reasons to believe that the experimental channel sounder used by Microcell Solutions for the channel measurements suffers from large amounts of such design imperfections (see footnotes 19,24 , and 28 ). It is promising to note, though, that

\footnotetext{
${ }^{30}$ Note that each parameter in Table 2 varies differently from route to route without an apparent link with the variations of the other parameters. Much more data is required to establish possible correlations.
} 


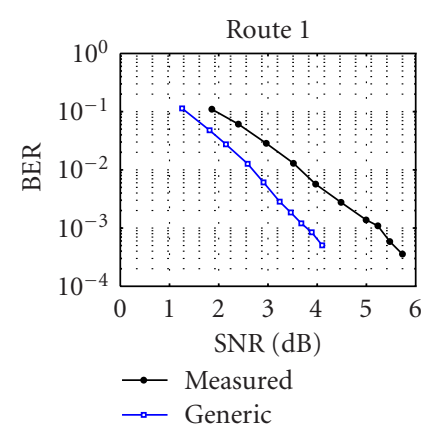

(a)

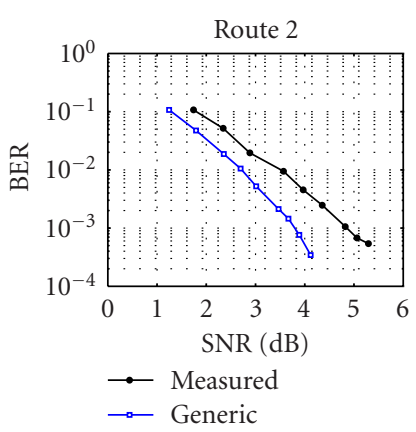

(b)

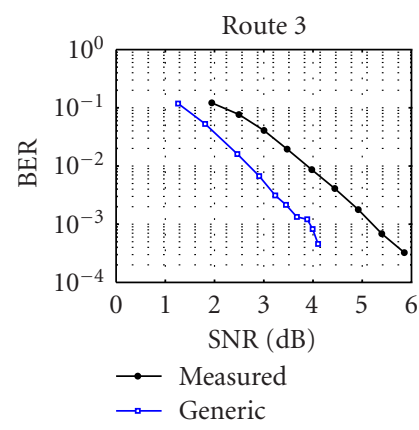

(c)

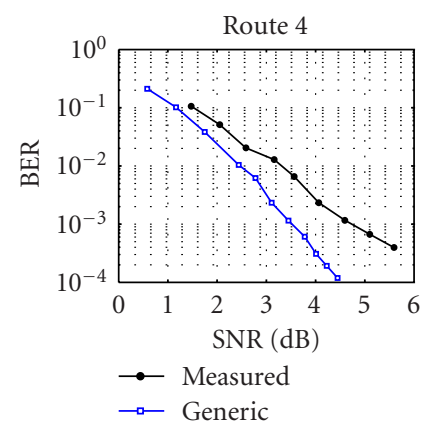

(d)

FIGURE 9: Link-level performance of STAR at $8 \mathrm{kbps}$ for both measured and generic channels: BER versus input SNR with one receive antenna.

TABLE 3: System-level performance of STAR at $8 \mathrm{kbps}$ for both measured and generic channels: required SNR at $10^{-3}$ BER and corresponding capacity at $1 \%$ outage with one receive antenna.

\begin{tabular}{|c|c|c|c|c|c|c|c|c|}
\hline \multirow[b]{2}{*}{ Channel } & \multicolumn{2}{|c|}{ Route 1} & \multicolumn{2}{|c|}{ Route 2} & \multicolumn{2}{|c|}{ Route 3} & \multicolumn{2}{|c|}{ Route 4} \\
\hline & $\begin{array}{l}\mathrm{SNR}_{\text {req }} \\
(\mathrm{dB})\end{array}$ & $\begin{array}{c}C_{\max } \\
\text { (users per cell) }\end{array}$ & $\begin{array}{l}\mathrm{SNR}_{\text {req }} \\
(\mathrm{dB})\end{array}$ & $\begin{array}{c}\quad C_{\max } \\
\text { (users per cell) }\end{array}$ & $\begin{array}{l}\mathrm{SNR}_{\text {req }} \\
(\mathrm{dB})\end{array}$ & $\begin{array}{c}\quad C_{\max } \\
\text { (users per cell) }\end{array}$ & $\begin{array}{l}\mathrm{SNR}_{\text {req }} \\
(\mathrm{dB})\end{array}$ & $\begin{array}{c}\quad C_{\max } \\
\text { (users per cell) }\end{array}$ \\
\hline Measured & 5.2 & 43 & 4.8 & 45 & 5.2 & 43 & 4.7 & 48 \\
\hline Generic & 3.9 & 63 & 3.8 & 63 & 4.0 & 58 & 3.6 & 64 \\
\hline Mismatch & 1.3 & $31 \%$ & 1.0 & $28 \%$ & 1.2 & $22 \%$ & 1.1 & $25 \%$ \\
\hline
\end{tabular}

such imperfection levels are unlikely in commercial wireless products. Lower SNR gaps and capacity losses could therefore be expected in practice. With the tested data, STAR (without a pilot, i.e., blind) would still accommodate about 45 DBPSK users per cell or sector at $8 \mathrm{kbps}$, thereby offering a potential spectrum efficiency of about $0.09 \mathrm{bps} / \mathrm{Hz}$ over measured channels with single transmit and receive antennas only.

\subsection{High data rate}

To verify the performance at higher data rates, we additionally considered the assessment of a HDR configuration of STAR at $512 \mathrm{kbps}$ before channel decoding (i.e., spreading factor $L=8$ ). To do so, we used the block-structured HDR implementation of STAR proposed in [12] with a processing block length of 256 chips (see details in [12]). Since reliable BER accuracy below $10^{-6}$ could not be achieved with the available data and simulation time increases significantly with the data rate, we limited the performance verification to the link-level only. System-level capacity results at this high rate will be considered in the next section along with the multiuser detection upgrade of STAR. Simulation results in terms of BER curves before channel decoding versus SNR in Figure 10 suggest that STAR works equally well at $512 \mathrm{kbps}$ over measured channels and that the SNR gap, assuming a target BER of $10 \%$ before channel decoding, is again in the range of $1 \mathrm{~dB}$ between measured and generic channels. This result is consistent with the observation made above on the impact of imperfection sources on performance as a main mismatch/loss factor.

\subsection{HDR with multiuser detection upgrade}

Multiuser detection and interference suppression are most effective for capacity enhancement at high data rates. Recently we proposed a new interference subspace rejection (ISR) paradigm $[4,8]$ as an upgrade of the simple MRC combiner of STAR. More recently, the resulting STAR-ISR receiver was found to outperform the RAKE-PIC (parallel interference canceller) by factor 4 to 7 in spectrum efficiency [12]. Here we further pushed the performance verification stage to the assessment of STAR-ISR at $512 \mathrm{kbps}$ over measured channels. To do so, we modified the linklevel module to include multiple access interference from a given number of in-cell (or in-sector) users as well as an equal number of STAR-ISR receivers dedicated each to the detection of one different user and to the suppression of the others. For each route, we sliced the corresponding channel recording into a similar number of far-spaced segments with equal durations. Each portion represents a shorter but quasiindependent channel of a different in-cell (or in-sector) user, taken however from the same route to keep the channel conditions identical for simplicity (i.e., the generic channels are tuned with the same set of parameters).

In Figure 11, the link-level simulation results in terms of required SNR at 10\% BER before channel decoding versus the number of in-cell (or in-sector) users suggest that 

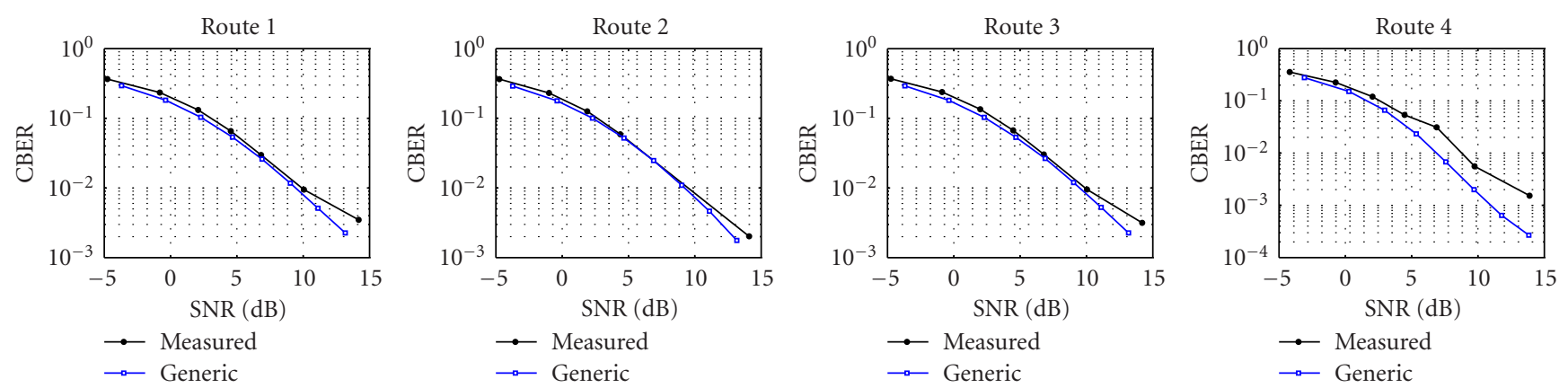

FIGURE 10: Link-level performance of STAR at $512 \mathrm{kbps}$ (i.e., $256 \mathrm{kbps}$ assuming rate-1/2 channel coding) for both measured and generic channels: CBER (coded BER, i.e., BER before channel decoding) versus input SNR with one receive antenna.
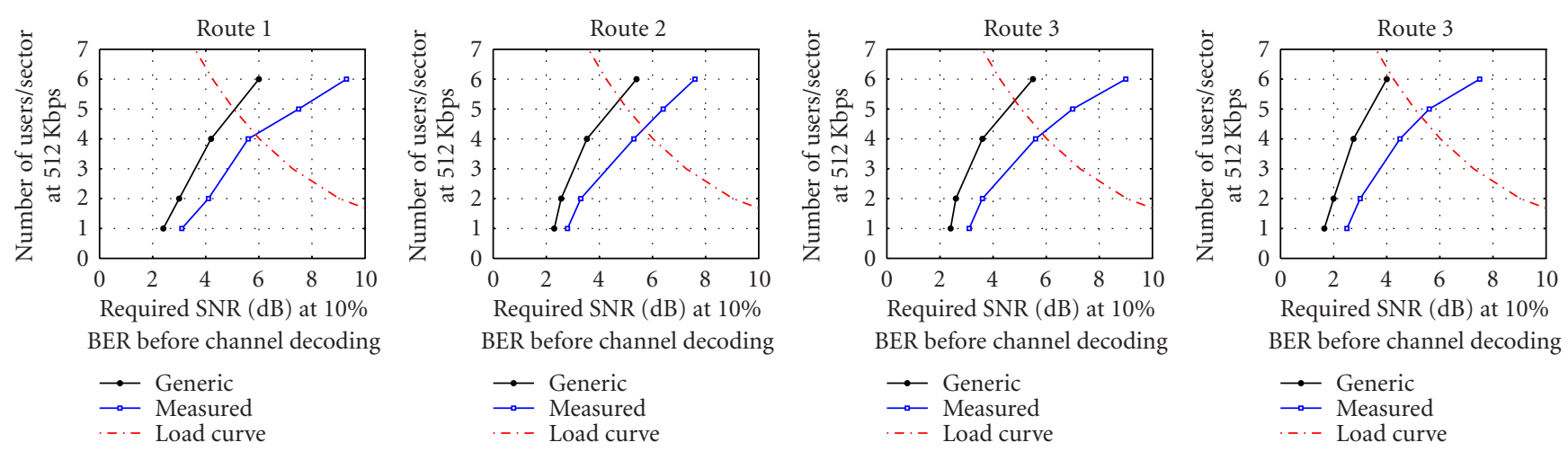

FIGURE 11: Link-level performance of STAR-ISR at $512 \mathrm{kbps}$ (i.e., $256 \mathrm{kbps}$ assuming rate-1/2 channel coding) for both measured and generic channels: required SNR at $10^{-1}$ BER (before channel decoding) versus the number of in-cell users per cell (or sector) targeted for suppression with one receive antenna (the load curve for an out-cell-to-in-cell interference ratio $f_{\mathrm{O} / \mathrm{I}}=0.6$ is in dashed line).

TABLE 4: System-level performance of STAR-ISR at $512 \mathrm{kbps}$ (i.e., $256 \mathrm{kbps}$ assuming rate-1/2 channel coding) for both measured and generic channels: required SNR at $10^{-1}$ BER (before channel decoding) and corresponding capacity (below load curve, see Figure 11) with one receive antenna.

\begin{tabular}{lcccccccc}
\hline & \multicolumn{2}{c}{ Route 1 } & \multicolumn{2}{c}{ Route 2 } & \multicolumn{2}{c}{ Route 3 } & \multicolumn{2}{c}{ Route 4 } \\
Channel & $\begin{array}{c}\mathrm{SNR}_{\text {req }} \\
(\mathrm{dB})\end{array}$ & $\begin{array}{c}C_{\text {max }} \\
\text { (users per cell) }\end{array}$ & $\begin{array}{c}\mathrm{SNR}_{\text {req }} \\
(\mathrm{dB})\end{array}$ & $\begin{array}{c}C_{\max } \\
\text { (users per cell) }\end{array}$ & $\begin{array}{c}\mathrm{SNR}_{\text {req }} \\
(\mathrm{dB})\end{array}$ & $\begin{array}{c}C_{\max } \\
\text { (users per cell) }\end{array}$ & $\begin{array}{c}\mathrm{SNR}_{\text {req }} \\
(\mathrm{dB})\end{array}$ & $\begin{array}{c}C_{\text {max }} \\
(\text { users per cell) }\end{array}$ \\
\hline Measured & 5.6 & 4 & 5.4 & 4 & 5.7 & 4 & 4.6 & 4 \\
Generic & 5.0 & 5 & 4.4 & 5 & 4.7 & 5 & 4.0 & 6 \\
Mismatch & 0.6 & $20 \%$ & 1.0 & $20 \%$ & 1.0 & $20 \%$ & 0.6 & $33 \%$ \\
\hline
\end{tabular}

the gap between the generic and measured channels keeps widening beyond the $1 \mathrm{~dB}$ range reported previously in the single-user case (i.e., the two lowest SNR points). With 6 users, the SNR gap is between 2 and $3.5 \mathrm{~dB}$. We attribute this widening gap to the larger impact of imperfections (not taken into account in the generic channels) on the reconstruction and suppression of increasing amounts of interference signals (see footnote 7). In any case, however, it is very important to report on the gap between the required SNR values at maximum achievable capacity for both the generic and measured channels. In Table 4 of system-level results, we provide these SNR values along with the corresponding maximum capacities achievable below the dashed-line load curve in Figure 11 for an out-cell-to-in-cell interference ratio $f_{\mathrm{O} / \mathrm{I}}=0.6$ [26]. As in the voice rate single-user case (cf. Section 5.1), the SNR gap is in the range of $1 \mathrm{~dB}$ and results in 20 to $30 \%$ losses in capacity. Yet, STAR-ISR (without a pilot, i.e., blind) would still accommodate 4 DBPSK users per cell or sector at $256 \mathrm{kbps}$ (assuming rate-1/2 channel coding), thereby offering a potential spectrum efficiency of $0.25 \mathrm{bps} / \mathrm{Hz}$ over measured channels with single transmit and receive antennas only. 


\subsection{Discussion}

In both cases of voice rate single-user STAR and HDR MUD STAR-ISR, imperfections not taken into account when using generic channels result in consistent shifts in required SNR of about $1 \mathrm{~dB}$. Although the associated capacity losses of 20 to $30 \%$ are significant, we still consider the performance achieved as promising, bearing in mind the fact that the experimental channel sounder used to collect the data does not match the accuracy of commercial wireless terminals because it was not initially intended to perform accurate and continuous recording (cf. Section 3.2). Lower losses in SNR performance could be anticipated in a prospective integration of STAR (or STAR-ISR) in wireless terminals.

Significant challenges remain, however, in verifying by means of field experiments with multiple moving transmitters and/or receivers the performance results obtained by simulation of the radio propagation environment. We are currently building a prototype of STAR to achieve its proof of concept "over the air." We have already laid out a preliminary architecture for STAR [27] based on hardware/software codesign. In a future step, we will integrate the STAR receiver in two MIMO hardware platforms, one for the mobile and one for the base station. This work provides hardware and software design guidelines and raises our confidence in the next design step, namely real-time software design for evaluation of real-time prototypes. Exploitation of the characteristics of generic channels has already led to significant complexity reduction $[2,27]$ and performance optimization [2]. The results reported verify the performance gains achievable with our channel analysis/synthesis approach to receiver operation.

\section{CONCLUSIONS}

In this work, we verified the analysis/synthesis-based design of STAR by illustrating its ability of instantly extracting the channel parameters (time delays and drifts, multipath components and average multipath power profiles, CFOs, Doppler spread or speed) from measured data and its ability of monitoring and adapting online to their time evolution in real-world propagation conditions. We also verified its performance by comparing the results achieved with generic and measured channels for an average multipath power profile of $[0,-4,-8] \mathrm{dB}$ and a vehicular speed of $30 \mathrm{~km} / \mathrm{h}$. With one transmit and one receive antenna only, results suggest that losses in SNR and capacity due to operation of STAR (without a pilot, i.e., blind) over measured channels are in the range of $1 \mathrm{~dB}$ and $20-30 \%$, respectively, with DBPSK. The required SNR threshold for receiver operation over measured channels is near $5 \mathrm{~dB}$. This corresponds to a spectrum efficiency of about $0.09 \mathrm{bps} / \mathrm{Hz}$ at $8 \mathrm{kbps}$, and $0.25 \mathrm{bps} / \mathrm{Hz}$ at $256 \mathrm{kbps}$ with a multiuser detection upgrade of STAR. Extensions to receivers with multiple transmit and receive antennas [8] can provide significant additional increases in spectrum efficiency.

\section{ACKNOWLEDGMENT}

This work was supported by a Canada Research Chair in High-Speed Wireless Communications and the NSERC Discovery Research Grants Program.

\section{REFERENCES}

[1] S. Affes and P. Mermelstein, "A new receiver structure for asynchronous CDMA: STAR-the spatio-temporal arrayreceiver," IEEE J. Select. Areas Commun., vol. 16, no. 8, pp. 1411-1422, 1998.

[2] K. Cheikhrouhou, S. Affes, and P. Mermelstein, "Impact of synchronization on performance of enhanced array-receivers in wideband CDMA networks," IEEE J. Select. Areas Commun., vol. 19, no. 12, pp. 2462-2476, 2001.

[3] S. Affes and P. Mermelstein, "Adaptive space-time processing for wireless CDMA," in Adaptive Signal Processing: Application to Real-World Problems, J. Benesty and Y. Huang, Eds., chapter 10, Springer-Verlag, Berlin, Germany, January 2003.

[4] S. Affes, H. Hansen, and P. Mermelstein, "Interference subspace rejection: a framework for multiuser detection in wideband CDMA," IEEE J. Select. Areas Commun., vol. 20, no. 2, pp. 287-302, 2002.

[5] S. Affes, J. Zhang, and P. Mermelstein, "Carrier frequency offset recovery for CDMA array-receivers in selective Rayleighfading channels," in Proc. IEEE 55th Vehicular Technology Conference (VTC'02), vol. 1, pp. 180-184, Birmingham, Ala, USA, May 2002.

[6] B. Smida, S. Affes, and P. Mermelstein, "Frequency and time synchronization for the CDMA array-receiver STAR with interference subspace rejection," in Proc. IEEE 58th Vehicular Technology Conference (VTC '03), vol. 2, pp. 887-891, Orlando, Fla, USA, October 2003.

[7] B. Smida, S. Affes, and P. Mermelstein, "Joint time-delay and frequency offset synchronization for CDMA array-receivers," in Proc. IEEE Signal Processing Advances in Wireless Communications (SPAWC '03), pp. 499-504, Rome, Italy, June 2003.

[8] H. Hansen, S. Affes, and P. Mermelstein, "High capacity downlink transmission with MIMO interference subspace rejection in multicellular CDMA networks," EURASIP Journal on Applied Signal Processing, vol. 2004, no. 5, pp. 707-726, 2004.

[9] G. L. Turin, F. D. Clapp, T. L. Johnston, S. B. Fine, and D. Lavry, "A statistical model of urban multipath," IEEE Trans. Veh. Technol., vol. 21, no. 1, pp. 1-9, 1972.

[10] H. Suzuki, "A statistical model for urban radio propagation," IEEE Trans. Commun., vol. 25, no. 7, pp. 673-680, 1977.

[11] J. Kivinen, X. Zhao, and P. Vainikainen, "Empirical characterization of wideband indoor radio channel at $5.3 \mathrm{GHz}$," IEEE Trans. Antennas Propagat., vol. 49, no. 8, pp. 1192-1203, 2001.

[12] S. Affes, K. Cheikhrouhou, and P. Mermelstein, "Enhanced interference suppression for spectrum-efficient high data-rate transmissions over wideband CDMA networks," in Proc. IEEE Int. Conf. Acoustics, Speech, Signal Processing (ICASSP '03), vol. 4, pp. 469-472, Hong Kong, China, April 2003.

[13] S. Affes, D. Feng, L. Ge, and P. Mermelstein, "Does directionof-arrival estimation help channel identification in multiantenna CDMA receivers?" in Proc. IEEE International Symposium on Advances in Wireless Communications (ISWC'02), pp. 167-168, Victoria, British Columbia, Canada, September 2002.

[14] 3rd Generation Partnership Project (3GPP), Technical Specification Group (TSG), Radio Access Network (RAN), Working Group (WG4), UE Radio Transmission and Reception (FDD), TS 25.101, V3.4.1, 2000. 
[15] 3rd Generation Partnership Project (3GPP), Technical Specification Group (TSG), Radio Access Network (RAN), Working Group (WG4), Base Station Conformance Testing (FDD), TS 25.141, V3.3.0, 2000.

[16] S. Aridhi, V. Roy, O. Fischer, B. Sultana, P. Marinier, and J. L. Gauvreau, "Third generation trial, final report-Phase 1," Tech. Rep., Microcell Connexions, Montreal, Canada, 1999.

[17] A. Jalali and P. Mermelstein, "Effects of diversity, power control, and bandwidth on the capacity of microcellular CDMA systems," IEEE J. Select. Areas Commun., vol. 12, no. 5, pp. 952-961, 1994.

[18] J. G. Proakis, Digital Communications, McGraw-Hill, New York, NY, USA, 3rd edition, 1995.

[19] K. Cheikhrouhou, S. Affes, and P. Mermelstein, "Robustness of enhanced STAR to multipath power profile variations in wideband CDMA channels," in Proc. 21st Biennial Symposium on Communications, pp. 250-254, Queen's University, Kingston, Ontario, Canada, June 2002.

[20] 3rd Generation Partnership Project, Technical Specification Group Radio Access Networks, UTRA (BS) FDD; Radio Transmission and reception, 3GPP TS 25.104, V4.1.0, 2001.

[21] C. Tepedelenlioglu, A. Abdi, G. B. Giannakis, and M. Kaveh, "Estimation of Doppler spread and signal strength in mobile communications with applications to handoff and adaptive transmission," Wireless Communications and Mobile Computing, vol. 1, no. 2, pp. 221-242, 2001.

[22] H. Hansen, S. Affes, and P. Mermelstein, "A Rayleigh Doppler frequency estimator derived from maximum likelihood theory," in Proc. 2nd IEEE Workshop on Signal Processing Advances in Wireless Communications (SPAWC '99), pp. 382-386, Annapolis, Md, USA, May 1999.

[23] M. Ghogho, A. Swami, and T. Durrani, "Blind synchronization and Doppler spread estimation of MSK signals in the context of time-selective fading channels," in Proc. IEEE Int. Conf. Acoustics, Speech, Signal Processing (ICASSP '00), vol. 5, pp. 2665-2668, Istambul, Turkey, June 2000.

[24] W. C. Jakes, Ed., Microwave Mobile Communications, John Wiley \& Sons, New York, NY, USA, 1974.

[25] R. H. Clarke and W. L. Khoo, "3-D mobile radio channel statistics," IEEE Trans. Veh. Technol., vol. 46, no. 3, pp. 798799, 1997.

[26] A. Viterbi, CDMA: Principles of Spread Spectrum Communications, Addison-Wesley, Reading, Mass, USA, 1995.

[27] S. Jomphe, J. Belzile, S. Affes, and K. Cheikhrouhou, "Codesign implementation of a 3G WCDMA base station receiver," in Proc. IEEE Canadian Conference on Electrical and Computer Engineering (CCECE '04), pp. 1191-1194, Niagara Falls, Ontario, Canada, May 2004.

Karim Cheikhrouhou received the Diplôme d'Ingénieur degree in telecommunications from the École Supérieure des Communications de Tunis in 1993, and the M.S. and Ph.D. degrees in electrical engineering and telecommunications from the École Nationale d'Ingénieurs de Tunis, Tunisia, in 1996 and 2004, respectively. From 1993 to 1998, he worked as a Research Engineer at the École Supérieure des Communications de Tunis, Tunisia, on switching systems and then on wireless channel characterization. Since March 2004, he has been a Postdoctoral Fellow at INRS-EMT, University of Quebec, Montreal, Canada. His research interests are in synchronization, wireless channel characterization, and antenna array receivers for wireless systems.
Sofiène Affes received the Diplôme d'Ingénieur degree in electrical engineering in 1992, and the Ph.D. degree with honors in signal processing in 1995, both from the École Nationale Supérieure des Télécommunications, Paris, France. He has been since then with INRS-EMT, University of Quebec, Montreal, Canada, as a Research Associate from 1995 till 1997, then as an Assistant Professor till 2000. Currently

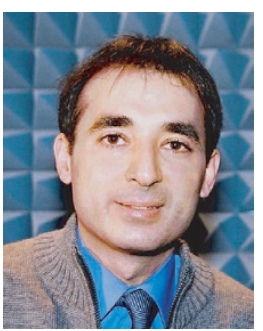
he is an Associate Professor in the Personal Communications Group. His research interests are in wireless communications, statistical signal and array processing, synchronization, multiuser detection, and MIMO systems. In 1997 he participated in the major program in personal and mobile communications of the Canadian Institute for Telecommunications Research. From 1998 to 2001 he has been leading the radio-design and signal processing activities of the Bell/Nortel/NSERC Industrial Research Chair in Personal Communications at INRS-EMT. Professor Affes is the corecipient of the 2002 Prize for Research Excellence of INRS and currently holds a Canada Research Chair in high-speed wireless communications.

Ahmed Elderini received his B.S. degree in electrical engineering from Alexandria University, Egypt, in 1992, and the M.S. degree in telecommunications from INRSEMT, Montreal, Canada, in 2004. From 1993 to 1998, he was a telecommunication engineer for Shell Egypt responsible for the company's microwave networks used for phone calls and telemetry traffic transmission. From 1999 to 2000, he was hired by Ontario Hydro in Toronto, Ontario, Canada, as a wireless systems engineer to manage and improve the wireless coverage of the company's radio network. In 2000, he worked for Nortel Networks as an RF engineer in Montreal, Quebec, Canada, and in 2001 he filled the same position in BAE (formerly Marconi Canada). His research interests are in new transceiver designs for $3 \mathrm{G}$ and beyond.

Besma Smida received the Diplôme d'Ingénieur degree in telecommunications from École Supérieure des Communications de Tunis, Tunisia, in 1995, and the M.S. degree in telecommunications from INRS-EMT, University of Quebec, Montreal, Canada, in 1998. She is currently pursuing the Ph.D. degree in telecommunications at INRS-EMT. From 1998 to 1999, she worked as a Research Assistant in the Personal Communications Group, INRS-EMT, conducting research on speech coding. From 1999 to 2002, she was a Research Engineer in the Technology Evolution and Standards Group, Microcell Solutions, Montreal, surveying and studying radio-communication technology evolution. Her current research interests include multicarrier modulation, spread spectrum, antenna arrays, synchronization, and multiuser detection in wireless communication.

Paul Mermelstein received the B.Eng. degree in engineering physics from McGill University, Montreal, Canada, in 1959, and the S.M., E.E., and D.Sc. degrees in electrical engineering from the Massachusetts Institute of Technology, Cambridge, Mass, in 1960, 1963, and 1964, respectively. From 1964 to 1973, he was a member 
of the technical staff in the Speech and Communications Research Department, Bell Laboratories, Murray Hill, NJ, carrying out research studies on speech generation and recognition. From 1973 to 1977, he was with Haskins Laboratories, New Haven, Conn, conducting research on speech perception and recognition. $\mathrm{He}$ moved to Bell Northern Research, later Nortel Networks, in 1977 to lead a speech communications research group. In 1994 he moved to INRS-EMT, University of Quebec, as a Professor and the Bell/Nortel/NSERC Industrial Research Chair in Personal Communications. From 1994 to 2000, he was the leader of the major program in personal and mobile communications of the Canadian Institute for Telecommunications Research. He is a Fellow of the IEEE and the Acoustical Society of America and served as an Editor for Speech Communications of the IEEE Transactions on Communications.

Belhassen Sultana received the Diplôme d'Ingénieur degree in telecommunications from École Supérieure des Communications de Tunis, Tunisia, in 1995, and the M.S. degree in telecommunications from INRS-EMT, University of Quebec, Montreal, Canada, in 1998. He has been with Microcell Solutions, a Canadian GSM operator, since 1997. He was a system engineer in the Research \& Standard Group from 1997 to 1999 . He then occupied a long-term planning position in the BSS Planning Group from 1999 to 2002. Currently he is an RF engineer for the Radio Network Planning and Design Group. His interests are related to long-term planning for GSM/UMTS network implementations.

Venkatesh Sampath received his M.S. and Ph.D. degrees from Laval University, Quebec, Canada, after graduating from Bangalore University, India, all in electrical engineering. Dr. Sampath has over fifteen years of research and technical managerial experience in the academic and industrial worlds. $\mathrm{He}$ is currently working at Flarion Technologies, Bedminster, NJ, USA, in the Sys-

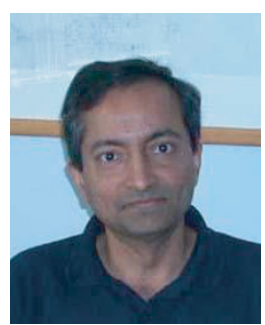
tems Test Group. Previously he worked as a

Consultant at Nextel Communications, Raleigh, NC, USA. From 1996 to 2002, Dr. Sampath worked at Microcell Connexions, the first fully digital GSM-based network operator in Canada. He was the Director of the Technology Evolution and Standards Group, which was responsible for R\&D-type activities (including standardisation). Prior to joining Microcell, he was a Research Associate at INRS-EMT, Montreal, Quebec, Canada, on radar- and PCS-related projects. His current interests are in designing qualification tests of the Flash-OFDM system, including base station and mobile terminals. 